TRANSACTIONS OF THE

AMERICAN MATHEMATICAL SOCIETY

Volume 360, Number 9, September 2008, Pages 4569-4595

S 0002-9947(08)04564-9

Article electronically published on April 10, 2008

\title{
STABLE REAL ALGEBRAIC VECTOR BUNDLES OVER A KLEIN BOTTLE
}

\author{
USHA N. BHOSLE AND INDRANIL BISWAS
}

\begin{abstract}
Let $X$ be a geometrically connected smooth projective curve of genus one, defined over the field of real numbers, such that $X$ does not have any real points. We classify the isomorphism classes of all stable real algebraic vector bundles over $X$.
\end{abstract}

\section{INTRODUCTION}

A Klein surface is a geometrically connected smooth projective curve, defined over the field of real numbers, satisfying the condition that it does not have any points defined over $\mathbb{R}[\mathrm{AG}$. For a geometrically connected smooth curve $X$ defined over $\mathbb{R}$, the corresponding complex curve $X_{\mathbb{C}}:=X \times_{\mathbb{R}} \mathbb{C}$ is equipped with an antiholomorphic involution $\sigma$ given by the involution of $\mathbb{C}$ defined by $z \longmapsto \bar{z}$. If $X$ does not have any points defined over $\mathbb{R}$, then $\sigma$ does not have any fixed points. Therefore, in that case the quotient space $X_{\mathbb{C}} / \sigma$ is a nonorientable topological surface. If, furthermore, $X$ is projective of genus one, then $X_{\mathbb{C}} / \sigma$ is evidently a Klein bottle.

By a Klein bottle we will mean a geometrically connected smooth projective curve of genus one defined over $\mathbb{R}$ that does not have any real points.

In At2, Atiyah classified the isomorphism classes of indecomposable vector bundles over a smooth elliptic curve defined over $\mathbb{C}$. His method can be generalized to classify indecomposable vector bundles on smooth elliptic curves defined over $\mathbb{R}$. By a smooth elliptic curve we mean a geometrically connected projective group of dimension one. Therefore, a smooth elliptic curve defined over $\mathbb{R}$ has an obvious point defined over $\mathbb{R}$, namely the identity element for the group law.

A Klein bottle does not have a real point, consequently it does not admit any real algebraic vector bundle of odd degree. On the other hand, when we consider real algebraic vector bundles of even degree, a Klein bottle has many more stable vector bundles than a smooth elliptic curve defined over $\mathbb{R}$. The space of stable holomorphic vector bundles of rank $r$ and degree $d$ over a smooth elliptic curve defined over $\mathbb{C}$ is empty if $\operatorname{gcd}(r, d) \neq 1$, and it is of complex dimension one if $\operatorname{gcd}(r, d)=1$. It follows from what we prove here that given any $r>0$ and $d$, the real dimension of the space of stable real algebraic vector bundles of rank $2 r$ and degree $2 d$ over a Klein bottle is two.

Received by the editors February 26, 2006.

2000 Mathematics Subject Classification. Primary 14H60; Secondary 14P99.

Key words and phrases. Stable bundle, real algebraic curve, Klein bottle.

(C)2008 American Mathematical Society

Reverts to public domain 28 years from publication 
Our aim here is to investigate the real algebraic vector bundles over a Klein bottle. We classify the isomorphism classes of real algebraic vector bundles of rank one and rank two over a Klein bottle. We also classify the isomorphism classes of stable real algebraic vector bundles of all ranks.

Take a Klein bottle $X$. For any integers $r^{\prime}$ and $d^{\prime}$, let $\mathcal{M}_{X_{\mathbb{C}}}\left(r^{\prime}, d^{\prime}\right)$ be the moduli space of stable vector bundles over $X_{\mathbb{C}}:=X \times_{\mathbb{R}} \mathbb{C}$ of rank $r^{\prime}$ and degree $d^{\prime}$. Let $\operatorname{Pic}^{0}\left(X_{\mathbb{C}}\right)$ be the Picard group parametrizing holomorphic line bundles over $X_{\mathbb{C}}$ of degree zero. The discrete subgroup of $\operatorname{Pic}^{0}\left(X_{\mathbb{C}}\right)$ defined by the line bundles of order $r^{\prime}$ will be denoted by $\Gamma_{r^{\prime}}$. Let $\operatorname{Pic}^{0}(X) \subset \operatorname{Pic}^{0}\left(X_{\mathbb{C}}\right)$ be the subgroup of real algebraic line bundles over $X$ of degree zero. Set

$$
\Gamma_{r^{\prime}}^{\mathbb{R}}:=\operatorname{Pic}^{0}(X) \cap \Gamma_{r^{\prime}} .
$$

Let $\sigma: X_{\mathbb{C}} \longrightarrow X_{\mathbb{C}}$ be the anti-holomorphic involution of the complexification $X_{\mathbb{C}}$. For a holomorphic vector bundle $F$ over $X_{\mathbb{C}}$, let $\bar{F}$ be the smooth complex vector bundle whose underlying real vector bundle is the one underlying the holomorphic vector bundle $F$, while the complex structure on the fibers of $\bar{F}$ is the conjugate complex structure of the fibers of $F$. The pullback $\sigma^{*} \bar{F}$ has a natural structure of a holomorphic vector bundle.

Given a real algebraic vector bundle $E$ on $X$, let $E_{\mathbb{C}}:=E \otimes_{\mathbb{R}} \mathbb{C}$ be the corresponding complex algebraic vector bundle over $X_{\mathbb{C}}$. We show that if $E$ is stable, then either $E_{\mathbb{C}}$ is stable, or $E_{\mathbb{C}}$ is isomorphic to $F \oplus \sigma^{*} \bar{F}$, where $F$ is a stable vector bundle over $X_{\mathbb{C}}$. Since the degree and rank of a stable vector bundle on $X_{\mathbb{C}}$ must be coprime, this implies that if $E$ is a stable real algebraic vector bundle over $X$ of rank $r$ and degree $d$, then either $r$ and $d$ are mutually coprime with $d$ even, or $\operatorname{gcd}(r, d)=2$; see Corollary 6.6. If $\operatorname{gcd}(r, d)=1$, then $E_{\mathbb{C}}$ is stable and the classification of $E$ in this case is similar to that of stable holomorphic vector bundles on $X_{\mathbb{C}}$. In the case $\operatorname{gcd}(r, d)=2$, one has $E_{\mathbb{C}} \cong F \oplus \sigma^{*} \bar{F}$, with $F$ stable. Thus the classification problem of stable vector bundles reduces to the problem of determining which $F \oplus \sigma^{*} \bar{F}$ are complexifications of real vector bundles on $X$; we solve the latter problem. Our main results may be summed up as follows:

- Assume that $\operatorname{gcd}(r, d)=1$ with $d$ an even integer and $r \geq 1$. Then the isomorphism classes of stable real algebraic vector bundles over $X$ of rank $r$ and degree $d$ are parametrized by $\operatorname{Pic}^{0}(X) / \Gamma_{r}^{\mathbb{R}}$ (see Theorem 6.3).

- If $\operatorname{gcd}(r, d)=2$ with $d=2 d^{\prime}$ and $r \geq 1$, where $d^{\prime}$ is an odd integer, then the isomorphism classes of stable real algebraic vector bundles over $X$ of rank $r$ and degree $d$ are canonically parametrized by the quotient space $\mathcal{M}_{X_{\mathbb{C}}}\left(\frac{r}{2}, d^{\prime}\right) /(\mathbb{Z} / 2 \mathbb{Z})$ for the involution of $\mathcal{M}_{X_{\mathbb{C}}}\left(\frac{r}{2}, d^{\prime}\right)$ defined by $W \longrightarrow$ $\sigma^{*} \bar{W}$. The space of isomorphism classes of stable real algebraic vector bundles over $X$ of rank $r$ and degree $d$ can be identified with the quotient space $\left(\operatorname{Pic}^{0}\left(X_{\mathbb{C}}\right) / \Gamma_{r / 2}\right) /(\mathbb{Z} / 2 \mathbb{Z})$ for the involution of $\operatorname{Pic}^{0}\left(X_{\mathbb{C}}\right) / \Gamma_{r / 2}$ defined by $L \longmapsto \sigma^{*} \bar{L}$ (see Proposition 6.7 and Corollary 6.8).

- Let $\operatorname{gcd}(r, d)=2$ with $r \geq 1$ and $d=2 d^{\prime}$, where $d^{\prime}$ is an even integer. Then the isomorphism classes of stable real algebraic vector bundles over $X$ of rank $r$ and degree $d$ are parametrized by the quotient by $\mathbb{Z} / 2 \mathbb{Z}$ of the complement $\left(\operatorname{Pic}^{0}\left(X_{\mathbb{C}}\right) / \Gamma_{r / 2}\right) \backslash\left(\operatorname{Pic}^{0}(X) / \Gamma_{r / 2}^{\mathbb{R}}\right)$ (see Corollary 6.10 and Proposition 6.9).

To classify the real algebraic vector bundles of rank two we first show that an indecomposable real algebraic vector bundle of rank 2 over $X$ is semi-stable. Let $E$ 
be an indecomposable real algebraic vector bundle over $X$ of rank two and degree zero. Then exactly one of the following two statements is valid:

(1) There is a real algebraic line bundle $\xi$ over $X$ of degree zero such that $E$ is obtained as the unique nontrivial extension of $\xi$ by itself.

(2) The vector bundle $E$ is stable.

The space of real algebraic vector bundles over $X$ of rank two and degree two are classified using the second statement in the above classification of stable vector bundles.

We will give a brief description of the sections. In Section 2 we recall the classification of Klein bottles. We show that the real algebraic vector bundle on a Klein bottle is uniquely determined by the corresponding vector bundle on the complex elliptic curve. In Section 3. we classify all the real algebraic line bundles on any given Klein bottle. In Section 4, we show that a real algebraic vector bundle over a Klein bottle decomposes into a direct sum of semistable vector bundles. In Section 5. we classify all the isomorphism classes of real algebraic vector bundles of rank two over a Klein bottle. In Section [6, we investigate the higher rank vector bundles over a Klein bottle. We classify all the isomorphism classes of polystable real algebraic vector bundles of rank at least three over a Klein bottle. While Section 6 is independent of Section 5 , it borrows heavily from the methods in Section 5 . In fact, Section 5 can be simplified using Lemma 6.5.

From our point of view it is difficult to parametrize the filtration data of semistable vector bundles which are not polystable. For this reason Section 6 falls short of classifying all real algebraic vector bundles over a Klein bottle.

\section{Preliminaries}

Let $X$ be a geometrically connected smooth projective curve of genus one defined over the field $\mathbb{R}$ of real numbers. We assume that $X$ does not have any points defined over $\mathbb{R}$. Such a curve is known as a Klein bottle $\mathrm{AG}$.

Let $X_{\mathbb{C}}=X \times_{\mathbb{R}} \mathbb{C}$ be the complex projective curve obtained by base change to $\mathbb{C}$. So $X_{\mathbb{C}}$ is an irreducible smooth curve of genus one.

The complex manifold $X_{\mathbb{C}}$ is equipped with an anti-holomorphic involution

$$
\sigma: X_{\mathbb{C}} \longrightarrow X_{\mathbb{C}},
$$

which is defined by the conjugation involution of field $\mathbb{C}$ that sends any $z$ to $\bar{z}$. Since $X$ does not have any points defined over $\mathbb{R}$, this involution $\sigma$ does not have any fixed points. Conversely, a smooth elliptic curve defined over $\mathbb{C}$, equipped with a fixed point free anti-holomorphic involution, gives a geometrically connected smooth projective curve of genus one, defined over $\mathbb{R}$, which does not have any points defined over $\mathbb{R}$. Therefore, by a Klein bottle we will also mean a smooth elliptic curve defined over $\mathbb{C}$ equipped with a fixed point free anti-holomorphic involution.

Let $V$ be a real algebraic vector bundle over the Klein bottle $X$. Let

$$
V_{\mathbb{C}}:=V \otimes_{\mathbb{R}} \mathbb{C}
$$

be the corresponding complex algebraic vector bundle over $X_{\mathbb{C}}$. Let $\overline{V_{\mathbb{C}}}$ be the $C^{\infty}$ complex vector bundle over $X_{\mathbb{C}}$ whose underlying real vector bundle is $V_{\mathbb{C}}$, and the complex structure of each fiber of $\overline{V_{\mathbb{C}}}$ is the conjugate of the complex structure of the fibers of $V_{\mathbb{C}}$. The vector bundle $\overline{V_{\mathbb{C}}}$ does not have a natural holomorphic structure. However, the vector bundle $\sigma^{*} \overline{V_{\mathbb{C}}}$ has a natural holomorphic - hence 
algebraic - structure, where $\sigma$ is the involution in eqn. 2.1). The fact that for any holomorphic function $f$ on $\mathbb{C}$, the function $z \longrightarrow \overline{f(\bar{z})}$ is also holomorphic ensures that $\sigma^{*} \overline{V_{\mathbb{C}}}$ has a natural holomorphic structure.

The involution $\sigma$ lifts to an algebraic isomorphism

$$
\delta: V_{\mathbb{C}} \longrightarrow \sigma^{*} \overline{V_{\mathbb{C}}}
$$

such that the composition

$$
V_{\mathbb{C}} \stackrel{\delta}{\longrightarrow} \sigma^{*} \overline{V_{\mathbb{C}}} \stackrel{\sigma^{*} \bar{\delta}}{\longrightarrow} \sigma^{*} \overline{\sigma^{*} \overline{V_{\mathbb{C}}}}=V_{\mathbb{C}}
$$

is the identity map of $V_{\mathbb{C}}$; since $\sigma^{2}=\operatorname{Id}_{X_{\mathbb{C}}}$, and $\overline{\bar{F}}=F$ for any complex vector bundle $F$, it follows that $\sigma^{*} \overline{\sigma^{*} \overline{V_{\mathbb{C}}}}$ is canonically identified with $V_{\mathbb{C}}$.

Let $W$ be another real algebraic vector bundle over $X$. Set $W_{\mathbb{C}}=W \otimes_{\mathbb{R}} \mathbb{C}$. Let

$$
\delta_{W}: W_{\mathbb{C}} \longrightarrow \sigma^{*} \overline{W_{\mathbb{C}}}
$$

be the isomorphism as in eqn. (2.2) for $W$. Then the real vector space $H^{0}(X, \operatorname{Hom}(V, W))$ is identified with the subspace of $H^{0}\left(X_{\mathbb{C}}, \operatorname{Hom}\left(V_{\mathbb{C}}, W_{\mathbb{C}}\right)\right)$ consisting of all homomorphisms $f$ such that the diagram

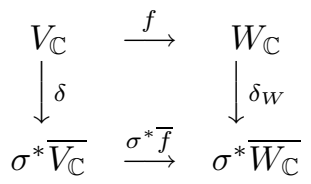

is commutative.

The following two categories are equivalent:

(1) the category of real algebraic vector bundles over $X$ with $\mathcal{O}_{X}$-linear homomorphisms as morphisms, and

(2) the category whose objects are pairs $(V, \delta)$, where $V$ is a holomorphic $(=$ algebraic) vector bundle over $X_{\mathbb{C}}$ and

$$
\delta: V \longrightarrow \sigma^{*} \bar{V}
$$

is an algebraic isomorphism, such that the composition $\left(\sigma^{*} \bar{\delta}\right) \circ \delta$ is the identity map of $V$ (see eqn. (2.3) ), and the morphisms from $(V, \delta)$ to $(F, \eta)$ are all $\mathcal{O}_{X_{\mathbb{C}}}$-linear homomorphisms

$$
\phi: V \longrightarrow F
$$

such that the diagram

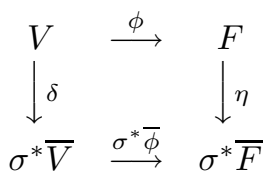

is commutative.

Lemma 2.1. Let $E_{1}$ and $E_{2}$ be two real algebraic vector bundles over a Klein bottle $X$. Let $V_{i}:=E_{i} \otimes_{\mathbb{R}} \mathbb{C}, i=1,2$, be the corresponding holomorphic vector bundles over $X_{\mathbb{C}}=X \times_{\mathbb{R}} \mathbb{C}$. If the two holomorphic vector bundles $V_{1}$ and $V_{2}$ are isomorphic, then $E_{1}$ is isomorphic to $E_{2}$. 
Proof. Assume that $V_{1}$ is isomorphic to $V_{2}$. The isomorphisms from $V_{1}$ to $V_{2}$ constitute a Zariski open dense subset

$$
\mathcal{U} \subset H^{0}\left(X_{\mathbb{C}}, V_{1}^{*} \otimes V_{2}\right) .
$$

For $i=1,2$, let

$$
\delta_{i}: V_{i} \longrightarrow \sigma^{*} \overline{V_{i}}
$$

be the isomorphism as in eqn. (2.2). We have a conjugate linear involution

$$
f: H^{0}\left(X_{\mathbb{C}}, V_{1}^{*} \otimes V_{2}\right) \longrightarrow H^{0}\left(X_{\mathbb{C}}, V_{1}^{*} \otimes V_{2}\right)
$$

defined by $T \longmapsto \delta_{2}^{-1} \circ\left(\sigma^{*} \bar{T}\right) \circ \delta_{1}$, where $T \in H^{0}\left(X_{\mathbb{C}}, V_{1}^{*} \otimes V_{2}\right)$.

Since $f$ is a conjugate linear involution, the subset

$$
\mathcal{S}:=\left\{\alpha \in H^{0}\left(X_{\mathbb{C}}, V_{1}^{*} \otimes V_{2}\right) \mid f(\alpha)=\alpha\right\} \subset H^{0}\left(X_{\mathbb{C}}, V_{1}^{*} \otimes V_{2}\right)
$$

is a totally real $\mathbb{R}$-linear subspace. In other words, we have

$$
H^{0}\left(X_{\mathbb{C}}, V_{1}^{*} \otimes V_{2}\right)=\mathcal{S} \oplus \sqrt{-1} \mathcal{S} .
$$

This implies that $\mathcal{S}$ is Zariski dense in $H^{0}\left(X_{\mathbb{C}}, V_{1}^{*} \otimes V_{2}\right)$.

Now it is easy to see that any

$$
T \in \mathcal{S} \cap \mathcal{U},
$$

where $\mathcal{U}$ is the Zariski open dense subset in eqn. (2.4), gives an isomorphism of $E_{1}$ with $E_{2}$. This completes the proof of the lemma.

We will now recall the classification of the Klein bottles.

Take any real number $\tau$, with $\tau>0$. Let

$$
\Lambda:=\{m \tau \sqrt{-1}+n \in \mathbb{C} \mid m, n \in \mathbb{Z}\}
$$

be the lattice in $\mathbb{C}$ generated by 1 and $\tau$. Let

$$
\widetilde{\sigma}: \mathbb{C} \longrightarrow \mathbb{C}
$$

be the map defined by $z \longmapsto \bar{z}+1 / 2$.

Let

$$
Y_{\tau}:=\mathbb{C} / \Lambda
$$

be the quotient space, which is an elliptic curve. The map $\widetilde{\sigma}$ in eqn. (2.6) descends to a self-map

$$
\sigma: Y_{\tau} \longrightarrow Y_{\tau}
$$

of the quotient space. It is easy to see that $\sigma$ is a fixed-point free anti-holomorphic involution of $Y_{\tau}$.

Therefore, the pair $\left(Y_{\tau}, \sigma\right)$ is a Klein bottle.

See [AG, p. 64, Theorem 1.9.8] for a proof of the following theorem.

Theorem 2.2. The isomorphism classes of Klein bottles, equivalently, the isomorphism classes of geometrically connected smooth projective curves of genus one, defined over $\mathbb{R}$, without ainy real points, are as follows: Given any Klein bottle $Y$, there is a unique real number $\tau>0$, such that the Klein bottle $\left(Y_{\tau}, \sigma\right)$ (see eqn. (2.7) and eqn. (2.8)) is isomorphic to $Y$. 
Remark 2.3. In [AG p. 64, Theorem 1.9.8], Klein bottles are classified as follows. For each elliptic curve $Y_{\tau}$ defined in eqn. (2.7), consider the fixed-point free antiholomorphic involution

$$
\sigma^{\prime}: Y_{\tau} \longrightarrow Y_{\tau}
$$

induced by the self-map

$$
z \longmapsto-\bar{z}+\frac{\sqrt{-1} \tau}{2}
$$

of $\mathbb{C}$. If $\tau>1$, then $\left(Y_{\tau}, \sigma\right)$ and $\left(Y_{\tau}, \sigma^{\prime}\right)$ are not isomorphic. For $\tau=1$, the two Klein bottles $\left(Y_{1}, \sigma\right)$ and $\left(Y_{1}, \sigma^{\prime}\right)$ are isomorphic by the isomorphism induced by the self-map of $\mathbb{C}$ defined by $z \longmapsto \sqrt{-1} z$. In [AG, Theorem 1.9.8] it is proved that any Klein bottle is isomorphic to exactly one Klein bottle from the union of the following three sets of Klein bottles:

(1) $\left(Y_{\tau}, \sigma\right)$, with $\tau>1$,

(2) $\left(Y_{\tau}, \sigma^{\prime}\right)$, with $\tau>1$, and

(3) $\left(Y_{1}, \sigma\right)$.

We note that $\left(Y_{\tau}, \sigma\right)$ is isomorphic to $\left(Y_{1 / \tau}, \sigma^{\prime}\right)$. To see this consider the isomorphism

$$
f_{\tau}: Y_{\tau} \longrightarrow Y_{1 / \tau}
$$

induced by the self-map of $\mathbb{C}$ defined by

$$
z \longmapsto \frac{\sqrt{-1} z}{\tau}
$$

It is easy to see that $\sigma=f_{\tau}^{-1} \circ \sigma^{\prime} \circ f_{\tau}$. Therefore, the two Klein bottles $\left(Y_{1}, \sigma\right)$ and $\left(Y_{1}, \sigma^{\prime}\right)$ are isomorphic. Therefore, Theorem 2.2 follows from Theorem 1.9.8 of $\mathrm{AG}$.

In the next section we will describe the real algebraic line bundles over a Klein bottle.

\section{Line BUndLes of the Klein Bottle}

Let $(Y, \sigma)$ be a Klein bottle. For any integer $d$, let $\operatorname{Pic}^{d}(Y)$ denote the Picard variety parametrizing isomorphism classes of holomorphic line bundles of degree $d$ over the compact connected Riemann surface $Y$. Let $Y_{\mathbb{R}}$ be the smooth projective curve defined over $\mathbb{R}$ corresponding to $(Y, \sigma)$. Therefore, $Y=Y_{\mathbb{R}} \times_{\mathbb{R}} \mathbb{C}$. The Picard variety $\operatorname{Pic}^{d}(Y)$ is the complexification of a variety defined over $\mathbb{R}$. To explain this, we note that the complex manifold $\operatorname{Pic}^{d}(Y)$ is equipped with an anti-holomorphic involution

$$
\sigma_{d}: \operatorname{Pic}^{d}(Y) \longrightarrow \operatorname{Pic}^{d}(Y)
$$

defined by $L \longmapsto \sigma^{*} \bar{L} \in \operatorname{Pic}^{d}(Y)$. Here $\bar{L}$ is the smooth complex line bundle over $Y$ whose underlying real vector bundle of rank two is $L$, and the complex structure on the fibers of $\bar{L}$ is the conjugate complex structure of the fibers of $L$ (see Section 2). Since $\sigma$ is an anti-holomorphic map, the pull-back $\sigma^{*} \bar{L}$ has a natural holomorphic structure. The holomorphic structure of $\sigma^{*} \bar{L}$ is determined by the following condition. A smooth section of $\sigma^{*} \bar{L}$ over an analytic open subset $U \subset Y$ is holomorphic if the corresponding smooth section of $L$ over $\sigma(U)$ is holomorphic. Since $\sigma_{d}$ is an anti-holomorphic involution of $\operatorname{Pic}^{d}(Y)$, the pair $\left(\operatorname{Pic}^{d}(Y), \sigma_{d}\right)$ define a geometrically irreducible smooth projective variety defined over the field of real 
numbers. Consider the conjugate complex structure on the real manifold underlying the complex manifold $\operatorname{Pic}^{d}(Y)$ (if $J$ is the almost complex structure on $\operatorname{Pic}^{d}(Y)$, then the conjugate complex structure is $-J)$. The complex manifold defined by this conjugate complex structure is the twist of the complex variety $\operatorname{Pic}^{d}(Y)$ by the nontrivial element in the Galois group $\operatorname{Gal}((\mathbb{C} / \mathbb{R})=\mathbb{Z} / 2 \mathbb{Z}$. Consequently, the anti-holomorphic involution $\sigma_{d}$, which is actually an involutive holomorphic isomorphism between $\operatorname{Pic}^{d}(Y)$ and its Galois conjugate, defines a projective variety defined over $\mathbb{R}$. Hence the real points of the real algebraic variety $\left(\operatorname{Pic}^{d}(Y), \sigma_{d}\right)$ are parametrized by the fixed points of the involution $\sigma_{d}$. The complex variety given by the real variety $\left(\operatorname{Pic}^{d}(Y), \sigma_{d}\right)$ using the inclusion of $\mathbb{R}$ in $\mathbb{C}$ is identified with $\operatorname{Pic}^{d}(Y)$. (See [Si, Ch. I, $\S 1$ 1] and [Si, Ch. I, $\S 4$ ] for more details.)

Let $\operatorname{Pic}^{d}\left(Y_{\mathbb{R}}\right)$ denote the space of all real algebraic line bundles of degree $d$ over

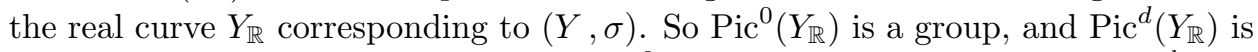
an affine space for it, which means that $\operatorname{Pic}^{0}\left(Y_{\mathbb{R}}\right)$ acts freely transitively on $\operatorname{Pic}^{d}\left(Y_{\mathbb{R}}\right)$. We note that this does not mean that $\operatorname{Pic}^{d}\left(Y_{\mathbb{R}}\right)$ is nonempty. If $\xi$ is a real algebraic line bundle over the curve $Y_{\mathbb{R}}$ corresponding to $(Y, \sigma)$, then we have

$$
\xi_{\mathbb{C}} \cong \sigma^{*} \overline{\xi_{\mathbb{C}}}
$$

where $\xi_{\mathbb{C}}=\xi \otimes_{\mathbb{R}} \mathbb{C}$ is the algebraic line bundle over $Y$ given by $\xi$. In particular, for any real algebraic line bundle $\xi$ over $Y_{\mathbb{R}}$ of degree $d$, the point in $\operatorname{Pic}^{d}(Y)$ corresponding to the line bundle $\xi \otimes_{\mathbb{R}} \mathbb{C}$ is defined over $\mathbb{R}$.

However, the converse is not true in general. A point of $\operatorname{Pic}^{d}(Y)$ defined over $\mathbb{R}$ need not correspond to a real algebraic line bundle over $Y_{\mathbb{R}}$. In fact, an algebraic line bundle $L \in \operatorname{Pic}^{d}(Y)$ corresponds to a real algebraic line bundle over the curve $Y_{\mathbb{R}}$ if and only if there is a holomorphic isomorphism

$$
\beta: L \longrightarrow \sigma^{*} \bar{L}
$$

such that the composition homomorphism

$$
L \stackrel{\beta}{\longrightarrow} \sigma^{*} \bar{L} \stackrel{\sigma^{*} \bar{\beta}}{\longrightarrow} \sigma^{*} \overline{\sigma^{*} \bar{L}}=L
$$

is the identity automorphism of $L$.

Proposition 3.1. For any integer $n$, the pair $\left(\operatorname{Pic}^{d}(Y), \sigma_{d}\right)$ is isomorphic to the pair $\left(\operatorname{Pic}^{d+2 n}(Y), \sigma_{d+2 n}\right)$.

For any integer $d$, the involution $\sigma_{2 d+1}$ of $\mathrm{Pic}^{2 d+1}(Y)$ does not have any fixed points.

For any integer $d$, there is a real algebraic line bundle of degree $2 d$ over the curve $Y_{\mathbb{R}}$.

Proof. Take any point $x_{0} \in Y$. Let $D=x_{0}+\sigma\left(x_{0}\right)$ be the divisor. The algebraic line bundle $\mathcal{O}_{Y}(D)$ over $Y$ defined by $D$ corresponds to a real algebraic line bundle over the curve $Y_{\mathbb{R}}$. Indeed,

$$
\sigma^{*} \overline{\mathcal{O}_{Y}(D)}=\mathcal{O}_{Y}(\sigma(D))=\mathcal{O}_{Y}(D) .
$$

Furthermore the tautological isomorphism $\beta: \mathcal{O}_{Y}(D) \longrightarrow \sigma^{*} \overline{\mathcal{O}_{Y}(D)}$ satisfies the condition that $\left(\sigma^{*} \bar{\beta}\right) \circ \beta=\operatorname{Id}_{\mathcal{O}_{Y}(D)}$.

Therefore, the morphism

$$
\operatorname{Pic}^{d}(Y) \longrightarrow \operatorname{Pic}^{d+2 n}(Y)
$$


defined by $L \longmapsto L \otimes \mathcal{O}_{Y}(n D)$ intertwines the involutions $\sigma_{d}$ and $\sigma_{d+2 n}$. Thus, the two pairs $\left(\operatorname{Pic}^{d}(Y), \sigma_{d}\right)$ and $\left(\operatorname{Pic}^{d+2 n}(Y), \sigma_{d+2 n}\right)$ are isomorphic.

To prove the second assertion, note that $\left(\operatorname{Pic}^{1}(Y), \sigma_{1}\right)$ is canonically identified with $(Y, \sigma)$ by sending any point $y \in Y$ to the line bundle $\mathcal{O}_{Y}(y)$. Since the involution $\sigma$ of $Y$ does not have any fixed points, the second assertion follows immediately.

Since the trivial holomorphic line bundle $\mathcal{O}_{Y}$ equipped with the smooth involution defined by $f \longmapsto \overline{f \circ \sigma}$, where $f$ is any locally defined holomorphic function on $Y$, satisfies the condition that the composition homomorphism as in eqn. (3.3) is the identity automorphism of $\mathcal{O}_{Y}$, the third assertion follows using the first assertion. Therefore, the proof of the proposition is complete.

To investigate the real algebraic variety defined by the degree zero line bundles over a Klein bottle, we will use the explicit description of a Klein bottle noted in Theorem 2.2 .

Take any positive real number $\tau$. Let $\left(Y_{\tau}, \sigma\right)$ be the corresponding Klein bottle (see eqn. (2.7) and eqn. (2.8)). For any $z \in \mathbb{C}$, the image of $z$ in the quotient $Y_{\tau}$ (see eqn. (2.7)) will be denoted by $\underline{z}$. We will identify $\operatorname{Pic}^{0}\left(Y_{\tau}\right)$ with $Y_{\tau}$ using the holomorphic map

$$
\phi: Y_{\tau} \longrightarrow \operatorname{Pic}^{0}\left(Y_{\tau}\right)
$$

defined by $x \longmapsto \mathcal{O}_{Y_{\tau}}(x-\underline{0})$. We note that $\phi$ does not intertwine the involution $\sigma$ of $Y_{\tau}$ and the involution $\sigma_{0}$ of $\operatorname{Pic}^{0}\left(Y_{\tau}\right)$ defined in eqn. (3.1). Indeed, the involution $\sigma$ does not have any fixed points, while $\sigma_{0}$ has fixed points, as shown in Proposition 3.1. We also note that $\phi$ is an isomorphism of algebraic groups.

The smooth projective curve defined over $\mathbb{R}$ corresponding to $\left(Y_{\tau}, \sigma\right)$ will be denoted by $Y_{\tau}^{\mathbb{R}}$.

With the above notation, we have the following theorem:

Theorem 3.2. Let $\operatorname{Pic}^{0}\left(Y_{\tau}\right)^{\sigma_{0}} \subset \operatorname{Pic}^{0}\left(Y_{\tau}\right)$ be the fixed point set for the involution $\sigma_{0}$ of $\operatorname{Pic}^{0}\left(Y_{\tau}\right)$.

(1) The fixed point set $\operatorname{Pic}^{0}\left(Y_{\tau}\right)^{\sigma_{0}}$ is the disjoint union

$\operatorname{Pic}^{0}\left(Y_{\tau}\right)^{\sigma_{0}}=\{\phi(\underline{r}) \mid r \in \mathbb{R} ; 0 \leq r<1\} \cup\{\phi(\underline{r+\sqrt{-1} \tau / 2}) \mid r \in \mathbb{R} ; 0 \leq r<1\}$.

(2) The subset $\{\phi(\underline{r}) \mid r \in \mathbb{R} ; 0 \leq r<1\} \subset \operatorname{Pic}^{0}\left(Y_{\tau}\right)^{\sigma_{0}}$ consists of all real algebraic line bundles over the curve $Y_{\tau}^{\mathbb{R}}$.

(3) For any holomorphic line bundle

$$
L \in\{\phi(\underline{r+\sqrt{-1} \tau / 2}) \mid r \in \mathbb{R} ; 0 \leq r<1\} \subset \operatorname{Pic}^{0}\left(Y_{\tau}\right)^{\sigma_{0}},
$$

there is no real algebraic line bundle $\xi$ over the curve $Y_{\tau}^{\mathbb{R}}$ such that $L=$ $\xi \otimes_{\mathbb{R}} \mathbb{C}$.

Proof. From the definition of $\phi$ we have

$$
\phi(\underline{z})=\mathcal{O}_{Y_{\tau}}(\underline{z}-\underline{0}) .
$$

Using this, from the definition of $\sigma$ in eqn. (2.8) we have

(3.7) $\sigma^{*} \overline{\phi(\underline{z})}=\mathcal{O}_{Y_{\tau}}(\underline{\widetilde{\sigma}(z)}-\underline{\tilde{\sigma}(0)})=\mathcal{O}_{Y_{\tau}}(\underline{\bar{z}+1 / 2}-\underline{1 / 2})=\phi(\underline{\bar{z}+1 / 2}) \otimes \phi(\underline{1 / 2})^{*}$

for all $z \in \mathbb{C}$, where $\widetilde{\sigma}$ is defined in eqn. (2.6). Since the holomorphic map

$$
\mathbb{C} \longrightarrow \operatorname{Pic}^{0}\left(Y_{\tau}\right)
$$


defined by $z \longmapsto \phi(\underline{z})$ is a group homomorphism, from eqn. (3.7) it follows that

$$
\sigma^{*} \overline{\phi(\underline{z})}=\phi(\underline{\bar{z}}) .
$$

Now fix any $z \in \mathbb{C}$ such that the following three conditions hold:

- $0 \leq \operatorname{Re}(z)<1$,

- $0 \leq \operatorname{Im}(z)<\tau$, and

- for the algebraic group isomorphism $\phi$ defined in eqn. (3.4),

$$
\phi(\underline{z}) \cong \sigma^{*} \overline{\phi(\underline{z})},
$$

where $\sigma$ is defined in eqn. (2.8), and $\operatorname{Re}(z)$ (respectively, $\operatorname{Im}(z)$ ) is the real (respectively, imaginary) part of $z$.

We recall that a line bundle $L \in \operatorname{Pic}^{0}\left(Y_{\tau}\right)$ is a fixed point of the involution $\sigma_{0}$ if and only if $L \cong \sigma^{*} \bar{L}$. Now, from eqn. (3.8) and eqn. (3.9) we have

$$
z-\bar{z} \in \Lambda \text {, }
$$

where $\Lambda$ is the lattice in (2.5).

As $0 \leq \operatorname{Im}(z)<\tau$, from eqn. (3.10) we conclude that the equality in eqn. (3.5) is valid. This proves the first statement in the theorem.

To prove the second statement, let $I$ denote the closed interval $[0,1 / 2] \subset \mathbb{R}$. Consider the map

$$
\widetilde{\gamma}: \mathbb{C} \times I \longrightarrow \mathbb{C}
$$

defined by $\widetilde{\gamma}(z, t)=z+t$. This map $\widetilde{\gamma}$ induces a map

$$
\gamma: Y_{\tau} \times I \longrightarrow Y_{\tau} \text {. }
$$

For any $t \in I$, let

$$
\gamma_{t}: Y_{\tau} \longrightarrow Y_{\tau}
$$

be the map defined by $z \longmapsto \gamma((z, t))$.

A flat connection on a holomorphic line bundle is said to be compatible with the holomorphic structure if every locally defined flat section is holomorphic. If $L$ is a holomorphic line bundle over $Y_{\tau}$ equipped with a flat connection compatible with the holomorphic structure, then parallel translations along $\gamma$ (defined in eqn. (3.11) ) give a holomorphic isomorphism of the holomorphic line bundle $L$ with $\gamma_{1 / 2}^{*} L$.

Take any $z_{0} \in \mathbb{C}$. We will give an explicit description of the holomorphic line bundle $\phi\left(\underline{z_{0}}\right)$ over $Y_{\tau}$.

Consider the holomorphically trivial line bundle

$$
\xi_{0}=Y_{\tau} \times \mathbb{C}
$$

over $Y_{\tau}$ with fiber $\mathbb{C}$. In other words, the sheaf of holomorphic sections of $\xi_{0}$ is the sheaf of holomorphic functions on $Y_{\tau}$. Therefore, the Dolbeault operator on $\xi_{0}$ defining its holomorphic structure is simply the differential that sends any locally defined complex valued smooth function $f$ to $\bar{\partial} f$. This line bundle $\xi_{0}$ is equipped with the Hermitian structure defined by $|(y, c)|^{2}=c \bar{c}$, where $y \in Y_{\tau}$ and $c \in \mathbb{C}$.

Let

$$
\omega:=\bar{\partial} z
$$

be the $(0,1)$-form on $Y_{\tau}=\mathbb{C} / \Lambda$ (here $z$ is the natural coordinate on the covering surface $\mathbb{C}$ of $\left.Y_{\tau}\right)$. Note that the $(0,1)$-form on $\mathbb{C}$ descends to the quotient $Y_{\tau}$. 
The holomorphic line bundle $\phi\left(\underline{z_{0}}\right)$ is the smooth line bundle $\xi_{0}$ equipped with the Dolbeault operator

$$
\bar{\partial}_{z_{0}}:=\bar{\partial}-\frac{\pi z_{0} \omega}{\tau}
$$

where $\omega$ is the form in eqn. (3.13). This expression for $\bar{\partial}_{z_{0}}$ can be deduced from the combination of the fact that the image of the line bundle $\phi\left(\underline{z_{0}}\right)$ in

$$
\operatorname{Pic}^{0}\left(Y_{\tau}\right)=\frac{H^{0}\left(Y_{\tau}, \Omega_{Y_{\tau}}\right)^{*}}{H_{1}\left(Y_{\tau}, \mathbb{Z}\right)}
$$

is given by integration of holomorphic one-forms on $Y_{\tau}$ along any smooth path connecting $\underline{z_{0}}$ to $\underline{0}$ and the fact that

$$
\int_{Y_{\tau}} \partial z \wedge \bar{\partial} \bar{z}=-2 \tau \sqrt{-1}
$$

Consider the connection

$$
D_{z_{0}}:=\partial+\frac{\pi \overline{z_{0} \omega}}{\tau}+\bar{\partial}_{z_{0}}
$$

on the line bundle $\phi\left(\underline{z_{0}}\right)$, where $\bar{\partial}_{z_{0}}$ is the Dolbeault operator in eqn. (3.14), and $\partial$ is the $(1,0)$-part of the de Rham differential. It is straightforward to check that $D_{z_{0}}$ (defined in eqn. (3.15) ) is a flat connection on $\phi\left(\underline{z_{0}}\right)$ compatible with the holomorphic structure.

In fact $D_{z_{0}}$ is the unique unitary flat connection on the holomorphic line bundle $\phi\left(\underline{z_{0}}\right)$, and a flat unitary metric on $\phi\left(\underline{z_{0}}\right)$ is given by the constant metric on the smooth trivial line bundle $Y_{\tau} \times_{\mathbb{R}} \mathbb{C}$ (recall that $\phi\left(z_{0}\right)$ was constructed by putting the Dolbeault operator $\bar{\partial}_{z_{0}}$ on the trivial line bundle). However, we will not need this for the proof.

Continuing with the proof of the theorem, for any $z_{0} \in \mathbb{C}$ we have

$$
\gamma_{1 / 2}^{*} \phi\left(\underline{z_{0}}\right)=\phi\left(\underline{z_{0}}\right),
$$

where $\gamma_{1 / 2}$ is defined in eqn. (3.12); note that

$$
\phi\left(\underline{z_{0}}\right)=\mathcal{O}_{Y_{\tau}}\left(\underline{z_{0}}-\underline{0}\right)
$$

and $\gamma_{1 / 2}^{*} \phi\left(\underline{z_{0}}\right)=\mathcal{O}_{Y_{\tau}}\left(\underline{z_{0}-1 / 2}-\underline{-1 / 2}\right)$.

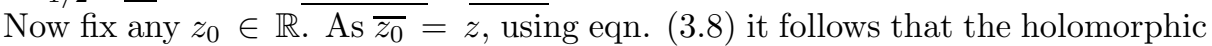
line bundle $\sigma^{*} \overline{\phi\left(\underline{z_{0}}\right)}$ is isomorphic to $\phi\left(\underline{z_{0}}\right)$. Therefore, the holomorphic line bundle $\gamma_{1 / 2}^{*} \phi\left(\underline{z_{0}}\right)$ is identified with the holomorphic line bundle $\sigma^{*} \overline{\phi\left(\underline{z_{0}}\right)}$. The parallel translations along $\gamma$ (defined in eqn. (3.11)) for the connection $D_{z_{0}}$ (defined in eqn. (3.15)) gives a holomorphic isomorphism of $\phi\left(\underline{z_{0}}\right)$ with $\sigma^{*} \overline{\phi\left(\underline{z_{0}}\right)}$. Let

$$
\eta: \phi\left(\underline{z_{0}}\right) \longrightarrow \sigma^{*} \overline{\phi\left(\underline{z_{0}}\right)}
$$

be the isomorphism obtained this way.

The composition

$$
\left(\sigma^{*} \bar{\eta}\right) \circ \eta: \phi\left(\underline{z_{0}}\right) \longrightarrow \sigma^{*} \overline{\sigma^{*} \overline{\phi\left(\underline{z_{0}}\right)}}=\phi\left(\underline{z_{0}}\right)
$$

is the holonomy of the connection $D_{z_{0}}$ (defined in eqn. (3.15)) along the closed path

$$
S^{1} \longrightarrow Y_{\tau}
$$


defined by $\exp (2 \pi \sqrt{-1} t) \longmapsto \underline{t}$, where $0 \leq t<1$. It is straightforward to check that $D_{z_{0}}$ has trivial holonomy along this loop.

Consequently, the composition $\left(\sigma^{*} \bar{\eta}\right) \circ \eta$ coincides with the identity map of $\phi\left(\underline{z_{0}}\right)$. Therefore, $\phi\left(\underline{z_{0}}\right)$ corresponds to a real algebraic line bundle over the real curve $\overline{Y_{\tau}^{\mathbb{R}}}$. This proves the second statement in the theorem.

To prove the third statement in the theorem, take any $z_{0}=r+\sqrt{-1} \tau / 2$, where $r \in \mathbb{R}$. Again we have

$$
\gamma_{1 / 2}^{*} \phi\left(\underline{z_{0}}\right) \cong \phi\left(\underline{z_{0}}\right),
$$

and the holomorphic line bundle $\gamma_{1 / 2}^{*} \phi\left(\underline{z_{0}}\right)$ is identified with $\sigma^{*} \overline{\phi\left(\underline{z_{0}}\right)}$. As before, let

$$
\eta: \phi\left(\underline{z_{0}}\right) \longrightarrow \sigma^{*} \overline{\phi\left(\underline{z_{0}}\right)}
$$

be the isomorphism given by parallel translation, for the connection $D_{z_{0}}$, along $\gamma$. It is straightforward to check that $D_{z_{0}}$ has holonomy -1 along the closed loop defined in eqn. (3.16). Therefore, the composition

$$
\left(\sigma^{*} \bar{\eta}\right) \circ \eta: \phi\left(\underline{z_{0}}\right) \longrightarrow \sigma^{*} \overline{\sigma^{*} \overline{\phi\left(\underline{\left.z_{0}\right)}\right.}}=\phi\left(\underline{z_{0}}\right)
$$

satisfies the identity

$$
\left(\sigma^{*} \bar{\eta}\right) \circ \eta=-\operatorname{Id}_{\phi}\left(\underline{z_{0}}\right) .
$$

If we replace the isomorphism $\eta$ by $\eta^{\prime}:=c \eta$, where $c \in \mathbb{C} \backslash\{0\}$, then using eqn. (3.17),

$$
\left(\sigma^{*} \bar{\eta}^{\prime}\right) \circ \eta^{\prime}=c \bar{c} \cdot \sigma^{*} \bar{\eta} \circ \eta=-c \bar{c} .
$$

Therefore, there is no isomorphism

$$
\eta^{\prime}: \phi\left(\underline{z_{0}}\right) \longrightarrow \sigma^{*} \overline{\phi\left(\underline{z_{0}}\right)}
$$

such that $\left(\sigma^{*} \overline{\eta^{\prime}}\right) \circ \eta^{\prime}=1$. This completes the proof of the theorem.

Combining Proposition 3.1 and Theorem 3.2, we obtain a complete classification of real algebraic line bundles on a Klein bottle. In particular, we have the following corollary.

Corollary 3.3. Let $X$ be a Klein bottle.

- The degree of any real algebraic line bundle over $X$ is an even integer.

- The space of real algebraic line bundles over $X$ of degree zero is parametrized by $\mathbb{R} / \mathbb{Z}$.

\section{Semistable vector Bundles and indecomposability}

Let $X$ be a Klein bottle, that is, a geometrically connected smooth projective curve of genus one, defined over $\mathbb{R}$, which does not have any points defined over $\mathbb{R}$. A real algebraic vector bundle $E$ over $X$ is called stable (respectively, semistable) if for all nonzero proper subbundles $F \subset E$, the inequality

$$
\frac{\operatorname{degree}(F)}{\operatorname{rank}(F)}<\frac{\operatorname{degree}(E)}{\operatorname{rank}(E)}
$$

(respectively, $\left.\frac{\operatorname{degree}(F)}{\operatorname{rank}(F)} \leq \frac{\operatorname{degree}(E)}{\operatorname{rank}(E)}\right)$ holds; see $[\mathrm{Ne}$ for more details. A semistable vector bundle over $X$ is called polystable if it is a direct sum of stable vector bundles. 
Lemma 4.1. Let $X_{\mathbb{C}}=X \times_{\mathbb{R}} \mathbb{C}$ be the corresponding complex elliptic curve. Let $E$ be a real algebraic vector bundle over $X$. Let $E_{\mathbb{C}}=E \otimes_{\mathbb{R}} \mathbb{C}$ be the corresponding vector bundle over $X_{\mathbb{C}}$.

(1) The vector bundle $E$ is semistable if and only if the vector bundle $E_{\mathbb{C}}$ over $X_{\mathbb{C}}$ is semistable.

(2) The vector bundle $E$ over $X$ splits into a direct sum of semistable vector bundles.

(3) The vector bundle $E$ is polystable if and only if $E_{\mathbb{C}}$ is polystable.

Proof. If $E_{\mathbb{C}}$ is semistable, then clearly $E$ is semistable. To prove the converse, assume that $E_{\mathbb{C}}$ is not semistable. Let

$$
0=V_{0} \subset V_{1} \subset V_{2} \subset \cdots \subset V_{\ell-1} \subset V_{\ell}=E_{\mathbb{C}}
$$

be the Harder-Narasimhan filtration of $E_{\mathbb{C}}$.

Let $\sigma$ be the anti-holomorphic involution of $X_{\mathbb{C}}$ as in eqn. (2.1). We have an isomorphism

$$
\delta: E_{\mathbb{C}} \longrightarrow \sigma^{*} \overline{E_{\mathbb{C}}}
$$

as in eqn. (2.2). Since both the filtrations

$$
0=\delta\left(V_{0}\right) \subset \delta\left(V_{1}\right) \subset \delta\left(V_{2}\right) \subset \cdots \subset \delta\left(V_{\ell-1}\right) \subset \delta\left(V_{\ell}\right)=\delta\left(E_{\mathbb{C}}\right)=\sigma^{*} \overline{E_{\mathbb{C}}}
$$

and

$$
0=\sigma^{*} \overline{V_{0}} \subset \sigma^{*} \overline{V_{1}} \subset \sigma^{*} \overline{V_{2}} \subset \cdots \subset \sigma^{*} \overline{V_{\ell-1}} \subset \sigma^{*} \overline{V_{\ell}}=\sigma^{*} \overline{E_{\mathbb{C}}}
$$

of $\sigma^{*} \overline{E_{\mathbb{C}}}$ satisfy the conditions of the Harder-Narasimhan filtration, from the uniqueness of the Harder-Narasimhan filtration we conclude that $\delta\left(V_{i}\right)=\sigma^{*} \overline{V_{i}}$ for all $i \in[1, \ell]$. Therefore, the subbundle $V_{i} \subset E_{\mathbb{C}}$ is given by a subbundle of $E$ by base change from $\mathbb{R}$ to $\mathbb{C}$. Hence the vector bundle $E$ is not semistable.

To prove the second statement in the lemma, we first note that if $V$ and $W$ are two semistable vector bundles over $X_{\mathbb{C}}$ with

$$
\frac{\operatorname{degree}(V)}{\operatorname{rank}(V)}>\frac{\operatorname{degree}(W)}{\operatorname{rank}(W)},
$$

then $H^{1}\left(X_{\mathbb{C}}, W^{*} \otimes V\right)=0$. Indeed, by Serre duality,

$$
H^{1}\left(X_{\mathbb{C}}, W^{*} \otimes V\right)^{*}=H^{0}\left(X_{\mathbb{C}}, W^{*} \otimes V \otimes K_{X_{\mathbb{C}}}\right) \cong H^{0}\left(X_{\mathbb{C}}, W^{*} \otimes V\right)=0
$$

as $K_{X_{\mathbb{C}}}$ is trivializable. Therefore, if $E$ over $X$ is not semistable, then the filtration in eqn. (4.1) splits completely. In other words, there is a filtration preserving isomorphism

$$
E_{\mathbb{C}} \longrightarrow \bigoplus_{i=1}^{\ell} \frac{V_{i}}{V_{i-1}} .
$$

We already noted that each $V_{i} / V_{i-1}$ corresponds to a real algebraic vector bundle over $X$. Therefore, the second statement follows from Lemma 2.1.

To prove the final statement in the lemma, take a polystable vector bundle $E$ over $X$. So, in particular, $E$ is semistable, and hence the corresponding vector bundle $E_{\mathbb{C}}$ over $X_{\mathbb{C}}$ is semistable. Assume that $E_{\mathbb{C}}$ is not polystable. Let

$$
W \subset E_{\mathbb{C}}
$$


be the socle of $E_{\mathbb{C}}$. In other words, $W$ is the unique maximal polystable subbundle of $E_{\mathbb{C}}$ with

$$
\frac{\operatorname{degree}(W)}{\operatorname{rank}(W)}=\frac{\operatorname{degree}\left(E_{\mathbb{C}}\right)}{\operatorname{rank}\left(E_{\mathbb{C}}\right)}
$$

(see [HL, p. 23, Lemma 1.5.5]).

Clearly, both the subbundles $\delta(W)$ and $\sigma^{*} \bar{W}$ of $\sigma^{*} \overline{\left(E_{\mathbb{C}}\right)}$ satisfy the conditions for a socle. From the uniqueness of the socle we conclude that that the two subbundles $\delta(W)$ and $\sigma^{*} \bar{W}$ coincide. Therefore, $W$ corresponds to a subbundle of $E$. Let $W_{\mathbb{R}} \subset E$ be the subbundle such that $W=W_{\mathbb{R}} \otimes_{\mathbb{R}} \mathbb{C}$.

Since $E$ is polystable, if $W_{\mathbb{R}} \neq E$, then there is another nonzero subbundle $W^{\prime} \subset E$ such that

$$
W_{\mathbb{R}} \oplus W^{\prime}=E .
$$

In that case, the socle of

$$
E_{\mathbb{C}}=\left(W_{\mathbb{R}} \otimes_{\mathbb{R}} \mathbb{C}\right) \oplus\left(W^{\prime} \otimes_{\mathbb{R}} \mathbb{C}\right)=W \oplus\left(W^{\prime} \otimes_{\mathbb{R}} \mathbb{C}\right)
$$

is $W \oplus W^{\prime \prime}$, where $W^{\prime \prime}$ is the socle of $W^{\prime} \otimes_{\mathbb{R}} \mathbb{C}$. But this contradicts the condition that $W$ is the socle of $E_{\mathbb{C}}$. Thus, $W_{\mathbb{R}}=E$. This completes the proof of the lemma.

The statement (2) in Lemma 4.1 has the following corollary:

Definition 4.2. A real algebraic vector bundle over $X$ is called decomposable if it splits into a direct sum of real algebraic vector bundles of positive ranks. A real algebraic vector bundle is called indecomposable if it is not decomposable.

Corollary 4.3. Any indecomposable real algebraic vector bundle over $X$ is semistable.

\section{Vector Bundles of RANK two over a Klein Bottle}

Let $X$ be a geometrically connected smooth projective curve of genus one, defined over $\mathbb{R}$, which does not have any points defined over $\mathbb{R}$. In this section we will describe the isomorphism classes of all real algebraic vector bundles of rank two over $X$.

We first recall that Proposition 3.1 says that for any even integer $n$, there is a real algebraic line bundle over $X$ of degree $n$. Also, in Theorem 3.2 and Proposition 3.1, all line bundles over $X$ are described. Therefore, it is enough to classify the isomorphism classes of indecomposable real algebraic vector bundles over $X$ of rank two and degree $d$, with $0 \leq d \leq 3$.

For any vector bundle $E$ over $X$ of rank two and degree $d$, we have

$$
d=\operatorname{degree}(E)=\operatorname{degree}\left(\bigwedge^{2} E\right) .
$$

Therefore, from Proposition 3.1 it follows that $d$ is an even integer. Thus, we need to consider the two cases of $d=0$ and $d=2$.

Let $X_{\mathbb{C}}:=X \times_{\mathbb{R}} \mathbb{C}$ be the smooth elliptic curve defined over $\mathbb{C}$. Let

$$
\sigma: X_{\mathbb{C}} \longrightarrow X_{\mathbb{C}}
$$

be the fixed point free anti-holomorphic involution as in eqn. (2.1) given by the conjugation of $\mathbb{C}$. 
Take any complex algebraic line bundle $L$ over $X_{\mathbb{C}}$. Consider the algebraic vector bundle

$$
S(L):=L \oplus \sigma^{*} \bar{L}
$$

of rank two over $X_{\mathbb{C}}$. For $S(L)$ we have

$$
\sigma^{*} \overline{S(L)}=\sigma^{*} \overline{L \oplus \sigma^{*} \bar{L}}=\sigma^{*} \bar{L} \oplus L .
$$

Therefore, there is a canonical isomorphism

$$
\sigma_{L}: S(L) \longrightarrow \sigma^{*} \overline{S(L)}
$$

defined by $\left(v_{1}, v_{2}\right) \longmapsto\left(v_{2}, v_{1}\right)$.

It is easy to check that the composition $\left(\sigma^{*} \overline{\sigma_{L}}\right) \circ \sigma_{L}$ is the identity automorphism of $S(L)$. Therefore, the pair $\left(S(L), \sigma_{L}\right)$ give a real algebraic vector bundle over $X$ of rank two (see Section 2). Let $V(L)$ denote the real algebraic vector bundle over $X$ of rank two given by $\left(S(L), \sigma_{L}\right)$.

Proposition 5.1. Let $L$ and $L^{\prime}$ be two complex algebraic line bundles over $X_{\mathbb{C}}$. Let $V(L)$ and $V\left(L^{\prime}\right)$ be the corresponding real algebraic vector bundles of rank two over $X$ (see the above construction). The two vector bundles $V(L)$ and $V\left(L^{\prime}\right)$ are isomorphic if and only if either $L$ is isomorphic to $L^{\prime}$ or $\sigma^{*} \bar{L}$ is isomorphic to $L^{\prime}$.

If degree $(L)=1$, then the above vector bundle $V(L)$ is stable.

Let $E$ be an indecomposable real algebraic vector bundle over $X$ of rank two and degree two. Then there is a complex algebraic line bundle $L$ of degree one over $X_{\mathbb{C}}$ such that the corresponding vector bundle $V(L)$ over $X$ is isomorphic to $E$.

Proof. First assume that $V(L) \cong V\left(L^{\prime}\right)$. So we have

$$
L \oplus \sigma^{*} \bar{L} \cong L^{\prime} \oplus \sigma^{*} \overline{L^{\prime}} .
$$

Now from [At1, p. 315, Theorem 2] it follows immediately that either $L \cong L^{\prime}$ or $\sigma^{*} \bar{L} \cong L^{\prime}$.

If $L \cong L^{\prime}$ or $\sigma^{*} \bar{L} \cong L^{\prime}$, then $S(L) \cong S\left(L^{\prime}\right)$, where $S(L)$ is defined in eqn. (5.2). If $S(L) \cong S\left(L^{\prime}\right)$, from Lemma 2.1 it follows that $V(L)$ and $V\left(L^{\prime}\right)$ are isomorphic. This completes the proof of the first part.

Now assume that degree $(L)=1$. Since $L \oplus \sigma^{*} \bar{L}$ is polystable, the vector bundle $V(L)$ is polystable (see Lemma 4.1(3)). As there are no line bundles over $X$ of degree one (see Proposition [3.1), the polystable vector bundle $V(L)$ of rank two and degree two must be stable.

We will now prove the final part of the proposition. Since $E$ is indecomposable, from Corollary 4.3 we know that $E$ is semistable.

We will show that $E$ is stable. To prove this, assume that $E$ is not stable. Therefore, there is a line subbundle

$$
\xi \subset E
$$

with degree $(\xi)=1$. But this contradicts the second assertion in Proposition 3.1 . Therefore, we conclude that the vector bundle $E$ is stable.

Since $E$ is stable, from Lemma 4.1(3) it follows immediately that the corresponding vector bundle

$$
E_{\mathbb{C}}:=E \otimes_{\mathbb{R}} \mathbb{C}
$$


over $X_{\mathbb{C}}$ is polystable. There are no stable vector bundles over $X_{\mathbb{C}}$ of rank two and degree two [At2, p. 428, Lemma 11]; see also Theorem 6.4 given in Section 6.2. Consequently,

$$
E_{\mathbb{C}}=L_{1} \oplus L_{2}
$$

with degree $\left(L_{1}\right)=1=\operatorname{degree}\left(L_{2}\right)$.

Let

$$
\delta: L_{1} \oplus L_{2}=E_{\mathbb{C}} \longrightarrow \sigma^{*} \overline{E_{\mathbb{C}}}=\sigma^{*} \overline{L_{1}} \oplus \sigma^{*} \overline{L_{2}}
$$

be the isomorphism corresponding to $E$ (see eqn. (2.2)). Now from [t1, p. 315, Theorem 2] it follows that either $L_{1} \cong \sigma^{*} \overline{L_{1}}$ or $L_{2} \cong \sigma^{*} \overline{L_{1}}$. On the other hand, since degree $\left(L_{1}\right)=1$, from the second statement in Proposition 3.1 it follows that $L_{1}$ is not isomorphic to $\sigma^{*} \overline{L_{1}}$. Thus, we conclude that

$$
L_{2} \cong \sigma^{*} \overline{L_{1}} \text {. }
$$

Therefore, $E_{\mathbb{C}} \cong L_{1} \oplus \sigma^{*} \overline{L_{1}}=S\left(L_{1}\right)$, where $S\left(L_{1}\right)$ is defined in eqn. (5.2). Now from Lemma 2.1 it follows that the real algebraic vector bundle $E$ over $X$ is isomorphic to $V\left(L_{1}\right)$. This completes the proof of the proposition.

Proposition 5.1 has the following corollary:

Corollary 5.2. The isomorphism classes of indecomposable real algebraic vector bundles over $X$ of rank two and degree two are parametrized by the quotient space

$$
X_{\mathbb{C}} / \sigma=\operatorname{Pic}^{1}\left(X_{\mathbb{C}}\right) / \sigma_{1},
$$

where $\sigma_{1}$ is the fixed point free involution defined by $L \longmapsto \sigma^{*} \bar{L}$.

Any indecomposable real algebraic vector bundle over $X$ of rank two and degree two is stable.

We will now investigate the indecomposable vector bundles over $X$ of rank two and degree zero.

Since the canonical line bundle of $X_{\mathbb{C}}$ is trivializable, from Lemma 2.1 it follows that the canonical line bundle $K_{X}$ of $X$ is also trivializable.

Let $\xi$ be a real algebraic line bundle over $X$. Since $K_{X}$ is trivializable, from Serre duality we have

$$
\operatorname{dim} H^{1}\left(X, \xi^{*} \otimes \xi\right)=\operatorname{dim} H^{0}\left(X, \mathcal{O}_{X}\right)=1 .
$$

Therefore, for $\xi$, there is a unique, up to an isomorphism, real algebraic vector bundle $W(\xi)$ over $X$ which is a nontrivial extension of $\xi$ by $\xi$. In other words, there is a unique, up to an isomorphism, indecomposable real algebraic vector bundle $W(\xi)$ over $X$ that fits in a short exact sequence

$$
0 \longrightarrow \xi \longrightarrow W(\xi) \longrightarrow \xi \longrightarrow 0 \text {. }
$$

Proposition 5.3. Let $E$ be a semistable real algebraic vector bundle over $X$ of rank two and degree zero. Then exactly one of the following three statements is valid:

(1) There is a real algebraic line bundle $\xi$ over $X$ of degree zero such that $W(\xi)$ is isomorphic to $E$ (the vector bundle $W(\xi)$ is defined in eqn. (5.5)).

(2) There are real algebraic line bundles $\xi_{1}$ and $\xi_{2}$ over $X$ of degree zero such that $\xi_{1} \oplus \xi_{2}$ is isomorphic to $E$.

(3) There is a line bundle $L \in \operatorname{Pic}^{0}\left(X_{\mathbb{C}}\right) \backslash \operatorname{Pic}^{0}(X)$ such that $V(L)$ is isomorphic to $E$, where $\operatorname{Pic}^{0}(X)$ denotes the group of line bundles of the form $L \otimes_{\mathbb{R}} \mathbb{C}$ with $L$ being a real algebraic line bundle over $X$ of degree zero, and $V(L)$ is defined prior to Proposition 5.1. 
For any $L \in \operatorname{Pic}^{0}\left(X_{\mathbb{C}}\right) \backslash \operatorname{Pic}^{0}(X)$, the real algebraic vector bundle $V(L)$ over $X$ is stable.

Proof. We will show that the above three cases correspond to following three mutually exclusive and exhaustive cases:

(1) $E$ is not polystable;

(2) $E$ is polystable, but not stable; and

(3) $E$ is stable.

The semistable vector bundle $E$ is not polystable if and only if it fits in a nonsplit short exact sequence

$$
0 \longrightarrow \xi \longrightarrow E \longrightarrow \eta \longrightarrow 0
$$

of real algebraic vector bundles over $X$, where degree $(\xi)=0=\operatorname{degree}(\eta)$. If $\eta \neq \xi$, then

$$
H^{1}\left(X, \eta^{*} \otimes \xi\right)=0
$$

and the above exact sequence splits. In other words, $\eta=\xi$ if $E$ is not polystable. Therefore, we conclude that $E \cong W(\xi)$ for some real algebraic line bundle $\xi$ over $X$ of degree zero if and only if $E$ is not polystable.

It is obvious that the semistable vector bundle $E$ is polystable but not stable if and only if $E \cong \xi_{1} \oplus \xi_{2}$, where $\xi_{1}$ and $\xi_{2}$ are some real algebraic line bundles of degree zero.

Take any line bundle $L \in \operatorname{Pic}^{0}\left(X_{\mathbb{C}}\right)$. We will show that $V(L)$ is stable if and only if $L \in \operatorname{Pic}^{0}\left(X_{\mathbb{C}}\right) \backslash \operatorname{Pic}^{0}(X)$.

Since the vector bundle $S(L)$ constructed in eqn. (5.2) is polystable, from Lemma 4.1(3) it follows that $V(L)$ is polystable. Now, $V(L)$ is not stable if and only if it decomposes as a direct sum of two line bundles. If $V(L)$ decomposes as a direct sum of two line bundles, from [At1, p. 315, Theorem 2] it follows that $L \in \operatorname{Pic}^{0}(X)$. Conversely, if $L \in \operatorname{Pic}^{0}(X)$, then $S(L)=L \oplus L$, as $L=\sigma^{*} \bar{L}$. Therefore, using Lemma 2.1 we conclude that for $L \in \operatorname{Pic}^{0}\left(X_{\mathbb{C}}\right)$, the vector bundle $V(L)$ is isomorphic to a direct sum of two real algebraic line bundles over $X$ of degree zero.

Thus, the vector bundle $V(L)$ is stable if and only if $L \notin \operatorname{Pic}^{0}(X)$.

Let $E$ be a stable vector bundle over $X$ of rank two and degree zero. Then its complexification $E_{\mathbb{C}}$ (defined in eqn. (5.4)) is polystable (see Lemma 4.1(3)). In other words,

$$
E_{\mathbb{C}}=L_{1} \oplus L_{2}
$$

where $L_{i}, i=1,2$, are complex algebraic line bundles of degree zero over $X_{\mathbb{C}}$. Let

$$
\delta: L_{1} \oplus L_{2}=E_{\mathbb{C}} \longrightarrow \sigma^{*} \overline{E_{\mathbb{C}}}=\sigma^{*} \overline{L_{1}} \oplus \sigma^{*} \overline{L_{2}}
$$

be the isomorphism as in eqn. (2.2) corresponding to $E$. Since $E$ is stable, the image $\delta\left(L_{1}\right)$ is not contained in the subbundle $\sigma^{*} \overline{L_{1}} \subset \sigma^{*} \overline{E_{\mathbb{C}}}$. Consequently, $\delta$ induces a nonzero homomorphism from $L_{1}$ to $\sigma^{*} \overline{L_{2}}$. Since any nonzero homomorphism between two line bundles of degree zero is an isomorphism, we have $L_{1} \cong \sigma^{*} \overline{L_{2}}$.

Since $L_{1} \cong \sigma^{*} \overline{L_{2}}$, we have $S\left(L_{2}\right) \cong E_{\mathbb{C}}$, where $S\left(L_{2}\right)$ is defined in eqn. (5.2). Now from Lemma 2.1 it follows that $E \cong V\left(L_{2}\right)$.

We have already shown that if $L_{2} \in \operatorname{Pic}^{0}(X)$, then $V\left(L_{2}\right)$ is not stable. This completes the proof of the proposition.

The following proposition complements Proposition 5.3 . 
Proposition 5.4. Let $\xi_{1}$ and $\xi_{2}$ be real algebraic line bundles over $X$ of degree zero such that $W\left(\xi_{1}\right)$ is isomorphic $W\left(\xi_{2}\right)$. Then $\xi_{1}$ is isomorphic to $\xi_{2}$.

Let $\xi_{i}, i=1,2$, and $\xi_{i}^{\prime}, i=1,2$, be real algebraic line bundles over $X$ of degree zero such that

$$
\xi_{1} \oplus \xi_{2} \cong \xi_{1}^{\prime} \oplus \xi_{2}^{\prime} .
$$

Then either $\xi_{1} \cong \xi_{1}^{\prime}$ or $\xi_{1} \cong \xi_{2}^{\prime}$.

If $L_{1}, L_{2} \in \operatorname{Pic}^{0}\left(X_{\mathbb{C}}\right) \backslash \operatorname{Pic}^{0}(X)$ are line bundles such that $V\left(L_{1}\right)$ is isomorphic to $V\left(L_{2}\right)$, then either $L_{1} \cong L_{2}$ or $L_{1} \cong \sigma^{*} \overline{L_{2}}$.

Proof. Let

$$
f: W\left(\xi_{1}\right) \longrightarrow W\left(\xi_{2}\right)
$$

be an isomorphism. Consider the composition homomorphism

$$
\xi_{1} \hookrightarrow W\left(\xi_{1}\right) \stackrel{f}{\longrightarrow} W\left(\xi_{2}\right) \longrightarrow \xi_{2}
$$

(see eqn. (15.5)). Since any nonzero homomorphism $\xi_{1} \longrightarrow \xi_{2}$ is an isomorphism, and $W\left(\xi_{2}\right)$ is a nontrivial extension of $\xi_{2}$ by $\xi_{2}$, the above composition homomorphism must vanish identically. Hence $f$ induces an isomorphism of $\xi_{1}$ with $\xi_{2}$.

The second statement in the proposition follows from [At1, p. 315, Theorem 2] and Lemma 2.1.

The third statement in the proposition follows from [At1, p. 315, Theorem 2]. This completes the proof of the proposition.

Corollary 4.3 and Proposition 5.3 together have the following corollary:

Corollary 5.5. Let $E$ be an indecomposable real algebraic vector bundle over $X$ of rank two and degree zero. Then exactly one of the following two statements is valid:

(1) The vector bundle $E$ is not polystable. There is a real algebraic line bundle $\xi$ over $X$ of degree zero such that $W(\xi)$ is isomorphic to $E$ (the vector bundle $W(\xi)$ is defined in eqn. (5.5)).

(2) The vector bundle $E$ is stable. There is a line bundle $L \in \operatorname{Pic}^{0}\left(X_{\mathbb{C}}\right) \backslash$ $\operatorname{Pic}^{0}(X)$ such that $V(L)$ is isomorphic to $E$, where $\operatorname{Pic}^{0}(X)$ denotes the group of line bundles of the form $L \otimes_{\mathbb{R}} \mathbb{C}$ with $L$ being a real algebraic line bundle over $X$ of degree zero, and $V(L)$ is defined prior to Proposition [5.1.

Corollary 5.6. The isomorphism classes of stable real algebraic vector bundles over $X$ of rank two and degree zero are parametrized by the quotient space $\left(\operatorname{Pic}^{0}\left(X_{\mathbb{C}}\right) \backslash\right.$ $\left.\operatorname{Pic}^{0}(X)\right) / \sigma_{0}$, where $\sigma_{0}$ is the involution defined by $L \longmapsto \sigma^{*} \bar{L}$.

The isomorphism classes of polystable, but not stable, real algebraic vector bundles over $X$ of rank two and degree zero are parametrized by the quotient space $\left(\operatorname{Pic}^{0}(X) \times \operatorname{Pic}^{0}(X)\right) /(\mathbb{Z} / 2 \mathbb{Z})$ for the action of $\mathbb{Z} / 2 \mathbb{Z}$ that switches the factors.

The isomorphism classes of semistable, but not polystable, real algebraic vector bundles over $X$ of rank two and degree zero are parametrized by $\operatorname{Pic}^{0}(X)$.

Remark 5.7. Corollary 5.6 describes the isomorphism classes of indecomposable real algebraic vector bundles over $X$ of rank two and degree zero. Corollary $[5.2$ describes the isomorphism classes of indecomposable real algebraic vector bundles over $X$ of rank two and degree two. As was noted earlier, this gives a classification of all real algebraic vector bundles over $X$ of rank two. 


\section{Stable vector Bundles of Higher RANK}

In this section we will classify all stable real algebraic vector bundles over a Klein bottle. We will first investigate the case where the degree and the rank are mutually coprime.

6.1. Indecomposable bundles in coprime case. We begin by recalling a theorem from At2.

Fix a positive integer $r$. Take any integer $d$ such that $r$ and $d$ are mutually coprime. Let $Z$ be a smooth elliptic curve defined over $\mathbb{C}$. Let

$$
\Gamma_{r} \subset \operatorname{Pic}^{0}(Z)
$$

be the subgroup of line bundles $L$ over $Z$ such that $L^{\otimes r} \cong \mathcal{O}_{Z}$.

Since $r$ and $d$ are mutually coprime, from Lemma 4.1(2) it follows that an algebraic vector bundle $E$ over $Z$ of rank $r$ and degree $d$ is indecomposable if and only if $E$ is semistable (see the proof of Lemma 4.1 (2)), and it also follows that $E$ is semistable if and only if it is stable. The following theorem is a special case of At2, p. 442, Theorem 10].

Theorem 6.1 (At2). Let $\mathcal{M}_{Z}(r, d)$ denote the moduli space of stable vector bundles over $Z$ of rank $r$ and degree $d$, where $r$ and $d$ are mutually coprime. Then $\mathcal{M}_{Z}(r, d)$ is nonempty. Take any stable vector bundle $E \in \mathcal{M}_{Z}(r, d)$, and consider the morphism

$$
\gamma_{E}: \operatorname{Pic}^{0}(Z) \longrightarrow \mathcal{M}_{Z}(r, d)
$$

defined by $L \longmapsto E \otimes L$. Then $\gamma_{E}$ is a surjective étale Galois covering. Furthermore,

$$
\gamma_{E}^{-1}(E)=\Gamma_{r}
$$

where $\Gamma_{r}$ is defined in eqn. (6.1). Therefore, $\Gamma_{r}$ is the Galois group for the covering $\gamma_{E}$.

Let $\left(Y_{\tau}, \sigma\right)$ be a Klein bottle defined as in eqn. (2.7) and eqn. (2.8). Let $X$ denote the geometrically connected smooth projective curve of genus one defined over $\mathbb{R}$ without any real points corresponding to $\left(Y_{\tau}, \sigma\right)$. Let $d$ be any even integer. As before, $r$ is a positive integer such that $r$ and $d$ are mutually coprime. We note that $r$ is an odd integer.

We will construct a stable real algebraic vector bundle over $X$ of rank $r$ and degree $d$. For that purpose, set

$$
\Lambda^{\prime}:=\{m \tau \sqrt{-1}+n r \in \mathbb{C} \mid m, n \in \mathbb{Z}\} .
$$

So $\Lambda^{\prime}$ is a sub-lattice of $\Lambda$ defined in eqn. (2.5). Therefore, we have a Galois covering map

$$
f: Y_{\tau}^{\prime}:=\mathbb{C} / \Lambda^{\prime} \longrightarrow \mathbb{C} / \Lambda=: Y_{\tau}
$$

with Galois group $\mathbb{Z} / r \mathbb{Z}$. Consider the anti-holomorphic involution

$$
\sigma^{\prime}: Y_{\tau}^{\prime} \longrightarrow Y_{\tau}^{\prime}
$$

of the quotient space $Y_{\tau}^{\prime}$ in eqn. (6.2) induced by the map $\mathbb{C} \longrightarrow \mathbb{C}$ defined by

$$
z \longmapsto \bar{z}+\frac{r}{2}
$$


Since $r$ is an odd integer, we have

$$
f \circ \sigma^{\prime}=\sigma \circ f,
$$

where $f$ is defined in eqn. (6.2) and $\sigma$ is defined in eqn. (2.8).

As $r$ is an odd integer, the involution $\sigma^{\prime}$ in eqn. (6.3) does not have any fixed points. Therefore, $\left(Y_{\tau}^{\prime}, \sigma^{\prime}\right)$ is a Klein bottle.

Fix a pair $\left(\xi, \beta^{\prime}\right)$, where $\xi$ is a holomorphic line bundle over $Y_{\tau}^{\prime}$ of degree $d$, and

$$
\beta^{\prime}: \xi \longrightarrow\left(\sigma^{\prime}\right)^{*} \bar{\xi}
$$

is an isomorphism as in eqn. (3.2) such that the composition homomorphism

$$
\xi \stackrel{\beta^{\prime}}{\longrightarrow}\left(\sigma^{\prime}\right)^{*} \bar{\xi} \stackrel{\left(\sigma^{\prime}\right)^{*} \overline{\beta^{\prime}}}{\longrightarrow}\left(\sigma^{\prime}\right)^{*} \overline{\left(\sigma^{\prime}\right)^{*} \bar{\xi}}=\xi
$$

is the identity automorphism of $\xi$; the map $\sigma^{\prime}$ is defined in eqn. (6.3). Since $d$ is an even integer, such a pair $\left(\xi, \beta^{\prime}\right)$ exist; see Proposition 3.1. Let $G$ denote the Galois group for the covering map $f$ in eqn. (6.2). Set

$$
\widehat{\xi}=\bigoplus_{\alpha \in G} \alpha^{*} \xi
$$

which is a holomorphic vector bundle over $Y_{\tau}^{\prime}$ of rank $r$ and degree $r d$.

The direct image $f_{*} \widehat{\xi}$ over $Y_{\tau}$ is equipped with an action of $G$. Let

$$
W:=\left(f_{*} \widehat{\xi}\right)^{G}
$$

be the invariant part of this action of $G$ on $f_{*} \widehat{\xi}$. It is easy to see that $W$ is a holomorphic vector bundle over $Y_{\tau}$ of rank $r$. Furthermore, the pullback $f^{*} W$ is canonically identified with $\widehat{\xi}$. This immediately implies that the degree of $W$ is $d$.

Since the vector bundle $\widehat{\xi}$ in eqn. (6.6) is polystable, and $f^{*} W=\widehat{\xi}$, we conclude that the vector bundle $W$ defined in eqn. (6.7) is also polystable.

Note that for any $\alpha \in G$, we have $\alpha \circ \sigma^{\prime}=\sigma^{\prime} \circ \alpha$. Consequently,

$$
\left(\sigma^{\prime}\right)^{*} \overline{\widehat{\xi}}=\bigoplus_{\alpha \in G} \alpha^{*}\left(\sigma^{\prime}\right)^{*} \bar{\xi} .
$$

Therefore, the isomorphism $\beta^{\prime}$ in eqn. (6.5) gives an isomorphism

$$
\widehat{\beta}^{\prime}:=\bigoplus_{\alpha \in G} \alpha^{*} \beta^{\prime}: \widehat{\xi}=\bigoplus_{\alpha \in G} \alpha^{*} \xi \longrightarrow \bigoplus_{\alpha \in G} \alpha^{*}\left(\sigma^{\prime}\right)^{*} \bar{\xi}=\left(\sigma^{\prime}\right)^{*} \overline{\widehat{\xi}} .
$$

Since $\left(\sigma^{\prime}\right)^{*} \overline{\beta^{\prime}} \circ \beta^{\prime}=\operatorname{Id}_{\xi}$, we have

$$
\left(\left(\sigma^{\prime}\right)^{*}{\widehat{\beta^{\prime}}}^{\prime} \circ \widehat{\beta}^{\prime}=\operatorname{Id}_{\widehat{\xi}} \cdot\right.
$$

Using eqn. (6.4), the isomorphism $\widehat{\beta}^{\prime}$ in eqn. (6.8) induces an isomorphism

$$
\widehat{\beta}: f_{*} \widehat{\xi} \longrightarrow \sigma^{*}\left(\overline{f_{*} \widehat{\xi}}\right),
$$

which commutes with the action of the group $G=\operatorname{Gal}(f)$ on $f_{*} \widehat{\xi}$. Therefore, $\widehat{\beta}$ induces an isomorphism

$$
\beta: W \longrightarrow \sigma^{*} \bar{W},
$$

where $W$ is defined in eqn. (6.7). From eqn. (6.9) it follows that $\left(\sigma^{*} \widehat{\widehat{\beta}}\right) \circ \widehat{\beta}=\operatorname{Id}_{f_{*} \widehat{\xi}}$, which in turn implies that

$$
\left(\sigma^{*} \bar{\beta}\right) \circ \beta=\operatorname{Id}_{W} .
$$


Therefore, the pair $(W, \beta)$ defines a real algebraic vector bundle over $X$ of rank $r$ and degree $d$, where $X$ is the real algebraic curve given by $\left(Y_{\tau}, \sigma\right)$. Let $W_{\mathbb{R}}$ denote this real algebraic vector bundle over $X$. We noted earlier that $W$ is polystable. Therefore, from Lemma 4.1(3) it follows that $W_{\mathbb{R}}$ is polystable. Since $r$ and $d$ are mutually coprime, we conclude that the polystable vector bundle $W_{\mathbb{R}}$ is stable.

We summarize the above construction in the following lemma:

Lemma 6.2. Let $X$ be a geometrically connected smooth projective curve of genus one, defined over $\mathbb{R}$, without any real points. Let $d$ be an even integer and $r$ a positive integer such that $r$ and $d$ are mutually coprime. Then there is a stable real algebraic vector bundle over $X$ of rank $r$ and degree $d$.

Let $\operatorname{Pic}^{0}(X)$ denote the group of real algebraic line bundles over $X$ of degree zero. From Theorem 3.2 we know that $\operatorname{Pic}^{0}(X)$ is identified with $\mathbb{R} / \mathbb{Z}$. The identification sends any $t \in \mathbb{R}$ to the line bundle corresponding to $\mathcal{O}_{Y_{\tau}}(\underline{t}-\underline{0}$ ) (as before, $\underline{z}$ is the image of $z \in \mathbb{C}$ in the quotient space $\left.Y_{\tau}=\mathbb{C} / \Lambda\right)$. Let

$$
\Gamma_{r}^{\mathbb{R}} \subset \operatorname{Pic}^{0}(X)
$$

be the subgroup of line bundles $L$ over $X$ whose order is a divisor of $r$, i.e., $L^{\otimes r} \cong$ $\mathcal{O}_{X}$.

Theorem 6.3. Let $X$ be a geometrically connected smooth projective curve of genus one, defined over $\mathbb{R}$, without any real points. Let $d$ be an even integer and $r$ a positive integer such that $r$ and $d$ are mutually coprime. Let $\mathcal{M}_{X}(r, d)$ denote the set of all isomorphism classes of stable real algebraic vector bundles over $X$ of rank $r$ and degree d. Fix $E \in \mathcal{M}_{X}(r, d)$ (it is nonempty by Lemma 6.2). Then the map

$$
\gamma: \operatorname{Pic}^{0}(X) \longrightarrow \mathcal{M}_{X}(r, d)
$$

defined by $L \longmapsto E \otimes L$ is surjective. Furthermore, $\gamma$ induces a bijection of $\mathcal{M}_{X}(r, d)$ with the quotient $\operatorname{Pic}^{0}(X) / \Gamma_{r}^{\mathbb{R}}$, where the subgroup $\Gamma_{r}^{\mathbb{R}}$ is defined in eqn. (6.12).

Proof. Since $r$ and $d$ are mutually coprime, if $F \in \mathcal{M}_{X}(r, d)$, then the corresponding vector bundle $F \otimes_{\mathbb{R}} \mathbb{C}$ over $Y_{\tau}$ is stable; see Lemma 4.1(3). Therefore, the set $\mathcal{M}_{X}(r, d)$ is in bijective correspondence with the isomorphism classes of pairs of the form $(W, \beta)$, where $W$ is a stable complex algebraic vector bundle over $Y_{\tau}$ of rank $r$ and degree $d$, and

$$
\beta: W \longrightarrow \sigma^{*} \bar{W}
$$

is an isomorphism as in eqn. (6.10) such that $\left(\sigma^{*} \bar{\beta}\right) \circ \beta=\operatorname{Id}_{W}$.

Let $\mathcal{M}_{Y_{\tau}}(r, d)$ denote the moduli space of stable vector bundles over $Y_{\tau}$ of rank $r$ and degree $d$. Since $r$ and $d$ are mutually coprime, the smooth variety $\mathcal{M}_{Y_{\tau}}(r, d)$ is projective. The variety $\mathcal{M}_{Y_{\tau}}(r, d)$ has an anti-holomorphic involution

$$
\delta_{(r, d)}: \mathcal{M}_{Y_{\tau}}(r, d) \longrightarrow \mathcal{M}_{Y_{\tau}}(r, d)
$$

defined by $V \longmapsto \sigma^{*} \bar{V}$. We note that $\mathcal{M}_{X}(r, d)$ is a subset of the fixed-point set of this involution $\delta_{(r, d)}$. As seen in Theorem 3.2. this subset of the fixed-point set is proper in general.

Let

$$
\delta_{(1,0)}: \operatorname{Pic}^{0}\left(Y_{\tau}\right) \longrightarrow \operatorname{Pic}^{0}\left(Y_{\tau}\right)
$$

be the anti-holomorphic involution defined by $L \longmapsto \sigma^{*} \bar{L}$. 
Fix any stable real algebraic vector bundle $E$ over $X$ of rank $r$ and degree $d$. Let $(W, \beta)$ be the pair corresponding to $E$, where $W$ is a complex algebraic vector bundle over $Y_{\tau}$ and $\beta$ is an isomorphism as in eqn. (6.10). Therefore, $W$ is isomorphic $\sigma^{*} \bar{W}$. Let

$$
\gamma_{W}: \operatorname{Pic}^{0}\left(Y_{\tau}\right) \longrightarrow \mathcal{M}_{Y_{\tau}}(r, d)
$$

be the morphism defined by $L \longmapsto W \otimes L$. From Theorem 6.1 we know that $\gamma_{W}$ is a surjective étale Galois covering with Galois group $\Gamma_{r}$, where

$$
\Gamma_{r} \subset \operatorname{Pic}\left(Y_{\tau}\right)
$$

is the subgroup defined by all line bundles $L$ such that $L^{\otimes r} \cong \mathcal{O}_{Y_{\tau}}$. Let

$$
\widetilde{\gamma_{W}}: \operatorname{Pic}^{0}\left(Y_{\tau}\right) / \Gamma_{r} \longrightarrow \mathcal{M}_{Y_{\tau}}(r, d)
$$

be the isomorphism given by $\gamma_{W}$ defined in eqn. (6.15) (see Theorem 6.1).

Since the subgroup $\Gamma_{r} \subset \operatorname{Pic}^{0}\left(Y_{\tau}\right)$ is preserved by the anti-holomorphic involution $\delta_{(1,0)}$ defined in eqn. (6.14), the involution $\delta_{(1,0)}$ descends to an antiholomorphic involution

$$
\delta: \operatorname{Pic}^{0}\left(Y_{\tau}\right) / \Gamma_{r} \longrightarrow \operatorname{Pic}^{0}\left(Y_{\tau}\right) / \Gamma_{r}
$$

of the quotient space.

As $W \cong \sigma^{*} \bar{W}$, we have

$$
\delta_{(r, d)} \circ \gamma_{W}=\gamma_{W} \circ \delta_{(1,0)},
$$

where $\delta_{(r, d)}$ (respectively, $\delta_{(1,0)}$ ) is defined in eqn. (6.13) (respectively, (6.14)), and $\gamma_{W}$ is defined in eqn. (6.15). This implies that

$$
\delta_{(r, d)} \circ \widetilde{\gamma_{W}}=\widetilde{\gamma_{W}} \circ \delta
$$

where $\widetilde{\gamma_{W}}$ is constructed in eqn. (6.16) and $\delta$ is constructed in eqn. (6.17).

Let

$$
q: \operatorname{Pic}^{0}\left(Y_{\tau}\right) \longrightarrow \operatorname{Pic}^{0}\left(Y_{\tau}\right) / \Gamma_{r}
$$

be the quotient map. Using the description of the fixed point set for the involution $\delta_{(1,0)}$ given in Theorem 3.2. together with the fact that $r$ is an odd integer, the following description of the fixed point set for the involution $\delta$ is obtained:

$$
\left(\operatorname{Pic}^{0}\left(Y_{\tau}\right) / \Gamma_{r}\right)^{\delta}=\{q(\phi(\underline{t})) \mid 0 \leq t<1 / r\} \cup\{q(\phi(\underline{t+\sqrt{-1} \tau / 2})) \mid 0 \leq t<1 / r\},
$$

where $\phi$ is defined in eqn. (3.4) and $q$ is defined above. From eqn. (6.18) we know that the fixed point set for the involution $\delta_{(r, d)}$ is the image

$$
\mathcal{M}_{Y_{\tau}}(r, d)^{\delta_{(r, d)}}=\widetilde{\gamma_{W}}\left(\left(\operatorname{Pic}^{0}\left(Y_{\tau}\right) / \Gamma_{r}\right)^{\delta}\right) .
$$

Take any $t \in \mathbb{R}$ such that $0 \leq t<1 / r$. The line bundle $\phi(\underline{t})$ over $Y_{\tau}$ has the property that there is an isomorphism

$$
\delta_{t}: \phi(\underline{t}) \longrightarrow \sigma^{*} \overline{\phi(\underline{t})}
$$

such that $\left(\sigma^{*} \overline{\delta_{t}}\right) \circ \delta_{t}=\operatorname{Id}_{\phi(\underline{\underline{t}})}$; see Theorem 3.2. The isomorphism

$$
\beta_{t}:=\beta \otimes \delta_{t}: W \otimes \phi(\underline{t}) \longrightarrow \sigma^{*} \bar{W} \otimes \sigma^{*} \overline{\phi(\underline{t})}=\sigma^{*}(\overline{W \otimes \phi(\underline{t})})
$$

satisfies the identity $\left(\sigma^{*} \overline{\beta_{t}}\right) \circ \beta_{t}=\operatorname{Id}_{W \otimes \phi(\underline{t})}$; recall that $\beta$ is the isomorphism corresponding to $E \in \mathcal{M}_{X}(r, d)$.

Therefore, $\gamma_{W}(\phi(\underline{t}))=W \otimes \phi(\underline{t})$ corresponds to a vector bundle in $\mathcal{M}_{X}(r, d)$. 
Now take $\mu=t+\sqrt{-1} \tau / 2$, where $0 \leq t<1 / r$. The line bundle $\phi(\mu)$ over $Y_{\tau}$ has the property that there is an isomorphism

$$
\delta_{\mu}^{\prime}: \phi(\underline{\mu}) \longrightarrow \sigma^{*} \overline{\phi(\underline{\mu})}
$$

such that $\left(\sigma^{*} \overline{\delta_{\mu}^{\prime}}\right) \circ \delta_{\mu}^{\prime}=-\operatorname{Id}_{\phi(\underline{\mu})}$; see eqn. (3.17). Consequently, the isomorphism

$$
\beta_{\mu}^{\prime}:=\beta \otimes \delta_{\mu}^{\prime}: W \otimes \phi(\underline{\mu}) \longrightarrow \sigma^{*} \overline{W \otimes \phi(\underline{\mu})}
$$

satisfies the identity $\left(\sigma^{*} \overline{\beta_{\mu}^{\prime}}\right) \circ \beta_{\mu}^{\prime}=-\operatorname{Id}_{W \otimes \phi(\underline{\mu})}$.

Since $W \otimes \phi(\mu)$ is stable, any isomorphism from $W \otimes \phi(\mu)$ to $\sigma^{*} \overline{W \otimes \phi(\mu)}$ must be of the form $\lambda \beta_{\mu}^{\prime}$ for some $\lambda \in \mathbb{C} \backslash\{0\}$. As

$$
\left(\sigma^{*} \overline{\lambda \beta_{\mu}^{\prime}}\right) \circ\left(\lambda \beta_{\mu}^{\prime}\right)=\lambda \bar{\lambda} \cdot\left(\sigma^{*} \overline{\beta_{\mu}^{\prime}}\right) \circ \beta_{\mu}^{\prime}=-|\lambda|^{2} \cdot \operatorname{Id}_{W \otimes \phi(\underline{\mu})} \neq 1,
$$

we conclude that there is no $E \in \mathcal{M}_{X}(r, d)$ such that $\gamma_{W}(\phi(\underline{\mu}))=W \otimes \phi(\underline{\mu})$ is isomorphic to $E \otimes_{\mathbb{R}} \mathbb{C}$. This completes the proof of the theorem.

6.2. Classification of stable vector bundles. We will now consider the general case where the rank and the degree are not necessarily mutually coprime. The following theorem of $[\mathrm{At2}$ and [Tu will be very useful.

Theorem 6.4. Let $V$ be a stable vector bundle over a smooth elliptic curve defined over $\mathbb{C}$. Then $\operatorname{rank}(V)$ and degree $(V)$ are mutually coprime.

See [Tu. p. 20, Appendix A] for a proof of Theorem 6.4

We note that for any Klein bottle $X$, there are stable vector bundles of rank two and degree two over $X$ (see Corollary [5.2), and also there are stable vector bundles of rank two and degree zero over $X$ (see Corollary 5.6).

Let $X$ be a geometrically connected smooth projective curve of genus one, defined over the real numbers, which does not have any points defined over $\mathbb{R}$. Let $\sigma$ be the anti-holomorphic involution of $X_{\mathbb{C}}=X \times_{\mathbb{R}} \mathbb{C}$ as in eqn. (2.1).

With the above notation, we have the following lemma:

Lemma 6.5. Let $V$ be a stable real algebraic vector bundle over $X$. Let

$$
V_{\mathbb{C}}:=V \otimes_{\mathbb{R}} \mathbb{C}
$$

be the corresponding complex algebraic vector bundle over $X_{\mathbb{C}}:=X \times_{\mathbb{R}} \mathbb{C}$. Then either $V_{\mathbb{C}}$ is stable, or $V_{\mathbb{C}}$ is isomorphic to $F \oplus \sigma^{*} \bar{F}$, where $F$ is a stable vector bundle over $X_{\mathbb{C}}$.

Proof. Let

$$
\delta: V_{\mathbb{C}} \longrightarrow \sigma^{*} \overline{V_{\mathbb{C}}}
$$

be the isomorphism as in eqn. (2.2). The vector bundle $V_{\mathbb{C}}$ is polystable, as $V$ is so (see Lemma 4.1(3)). Assume that $V_{\mathbb{C}}$ is not stable. Let

$$
V_{\mathbb{C}}=\bigoplus_{i=1}^{\ell} F_{i}
$$

be a decomposition of $V_{\mathbb{C}}$ into a direct sum of stable vector bundles.

Consider the holomorphic subbundle $\sigma^{*} \overline{F_{1}} \subset \sigma^{*} \overline{V_{\mathbb{C}}}$, where $F_{1}$ is the subbundle in eqn. (6.21). Let

$$
F^{\prime}:=\delta^{-1}\left(\sigma^{*} \overline{F_{1}}\right) \subset V_{\mathbb{C}}
$$


be the subbundle, where $\delta$ is the isomorphism in eqn. (6.20). Let

$$
\psi: F^{\prime} \longrightarrow V_{\mathbb{C}} / F_{1}
$$

be the natural projection.

Note that $V_{\mathbb{C}} / F_{1}$ is polystable, $F^{\prime}$ is stable, and

$$
\frac{\operatorname{degree}\left(V_{\mathbb{C}} / F_{1}\right)}{\operatorname{rank}\left(V_{\mathbb{C}} / F_{1}\right)}=\frac{\operatorname{degree}\left(F^{\prime}\right)}{\operatorname{rank}\left(F^{\prime}\right)} .
$$

Therefore, the above homomorphism $\psi$ is either the zero homomorphism, or it is a fiberwise injective homomorphism of vector bundles. If $\psi=0$, then $F^{\prime}=F_{1}$. In that case $F_{1}$ defines a real algebraic subbundle $F_{\mathbb{R}}$ of $V$ with

$$
\frac{\text { degree }\left(F_{\mathbb{R}}\right)}{\operatorname{rank}\left(F_{\mathbb{R}}\right)}=\frac{\text { degree }(V)}{\operatorname{rank}(V)} \text {. }
$$

But this contradicts the assumption that $V$ is stable.

Therefore, $\psi$ is a fiberwise injective homomorphism of vector bundles. This implies that $F_{1}+F^{\prime}$ is a subbundle of $V_{\mathbb{C}}$, and $F_{1}+F^{\prime}$ is identified with $F_{1} \oplus$ $F^{\prime}$; by $F_{1}+F^{\prime}$ we denote the coherent subsheaf of $V_{\mathbb{C}}$ generated by $F_{1}$ and $F^{\prime}$. Furthermore, the isomorphism $\delta$ takes the subbundle $F_{1} \oplus F^{\prime} \subset V_{\mathbb{C}}$ to the subbundle $\sigma^{*}\left(\overline{F_{1} \oplus F^{\prime}}\right) \subset \sigma^{*} \overline{V_{\mathbb{C}}}$. This follows from the fact that $\left(\sigma^{*} \bar{\delta}\right) \circ \delta=\operatorname{Id}_{V_{\mathbb{C}}}$. Therefore, $F_{1} \oplus F^{\prime}$ defines a real algebraic subbundle $W$ of $V$ with

$$
\frac{\operatorname{degree}(W)}{\operatorname{rank}(W)}=\frac{\text { degree }(V)}{\operatorname{rank}(V)} .
$$

Since $V$ is stable, from this we conclude that $F_{1} \oplus F^{\prime}=V_{\mathbb{C}}$. Since $F^{\prime}=\delta^{-1}\left(\sigma^{*} \overline{F_{1}}\right)$ is isomorphic to $\sigma^{*} \overline{F_{1}}$, the proof of the lemma is complete.

Theorem 6.4 and Lemma 6.5 have the following corollary:

Corollary 6.6. Let $E$ be a stable real algebraic vector bundle of rank $r$ and degree $d$ over a Klein bottle $X$. Then either $\operatorname{gcd}(r, d)=1$ or $\operatorname{gcd}(r, d)=2$.

Proposition 6.7. Let $X$ be a Klein bottle, and $X_{\mathbb{C}}:=X \times_{\mathbb{R}} \mathbb{C}$. Let $\sigma$ be the anti-holomorphic involution of $X_{\mathbb{C}}$. Fix a positive integer $r^{\prime}$ and an odd integer $d^{\prime}$ such that $r^{\prime}$ and $d^{\prime}$ are mutually coprime. Let $r:=2 r^{\prime}$ and $d:=2 d^{\prime}$.

(1) For any stable vector bundle $V$ over $X_{\mathbb{C}}$ of rank $r^{\prime}$ and degree $d^{\prime}$, the real algebraic vector bundle over $X$ defined by $V \oplus \sigma^{*} \bar{V}$ is stable.

(2) For any stable real algebraic vector bundle $E$ over $X$ of rank $r$ and degree $d$, there is a stable vector bundle $V$ over $X_{\mathbb{C}}$ of rank $r^{\prime}$ and degree $d^{\prime}$ such that

$$
E \otimes_{\mathbb{R}} \mathbb{C}=V \oplus \sigma^{*} \bar{V} .
$$

(3) Let $V$ and $W$ be stable vector bundles of rank $r^{\prime}$ and degree $d^{\prime}$ over $X_{\mathbb{C}}$, and let $V_{\mathbb{R}}$ (respectively, $W_{\mathbb{R}}$ ) be the real algebraic vector bundle over $X$ given by $V \oplus \sigma^{*} \bar{V}$ (respectively, $W \oplus \sigma^{*} \bar{W}$ ). Then $V_{\mathbb{R}}$ and $W_{\mathbb{R}}$ are isomorphic if and only if either $V$ is isomorphic to $W$ or $V$ is isomorphic to $\sigma^{*} \bar{W}$.

Proof. Let $V$ be a stable vector bundle over $X_{\mathbb{C}}$ of rank $r^{\prime}$ and degree $d^{\prime}$. Since $V \oplus \sigma^{*} \bar{V}$ is polystable, the real algebraic vector bundle $E$ over $X$ corresponding 
to $V \oplus \sigma^{*} \bar{V}$ is also polystable (see Lemma $4.1(3)$ ). Assume that $E$ is not stable. Therefore, there is a nonzero proper subbundle $E^{\prime} \subset E$ with

$$
\frac{\operatorname{degree}\left(E^{\prime}\right)}{\operatorname{rank}\left(E^{\prime}\right)}=\frac{d^{\prime}}{r^{\prime}} \text {. }
$$

Using eqn. 6.22 together with the conditions on $r^{\prime}$ and $d^{\prime}$ it follows that degree $\left(E^{\prime}\right)=d^{\prime}$. Since there are no real algebraic vector bundles over $X$ of odd degree (Proposition 3.1), and $d^{\prime}$ is an odd integer, we get a contradiction. Therefore, the vector bundle $E$ must be stable.

To prove the second statement, for any stable real algebraic vector bundle $E$ over $X$ of rank $r$ and degree $d$, consider the complex algebraic vector bundle $E_{\mathbb{C}}:=$ $E \otimes_{\mathbb{R}} \mathbb{C}$ over $X_{\mathbb{C}}$. From Lemma 4.1)(3) it follows that $E_{\mathbb{C}}$ is polystable, and from Theorem 6.4 it follows that $E_{\mathbb{C}}$ is not stable. Therefore, by Lemma 6.5, the vector bundle $E_{\mathbb{C}}$ is of the form $F \oplus \sigma^{*} \bar{F}$, where $F$ is a stable vector bundle over $X_{\mathbb{C}}$ of rank $r^{\prime}$ and degree $d^{\prime}$.

The third statement follows from [At1, p. 315, Theorem 2] and Lemma 2.1, This completes the proof of the proposition.

Let $\mathcal{M}_{X_{\mathbb{C}}}\left(r^{\prime}, d^{\prime}\right)$ be the moduli space of stable vector bundles over $X_{\mathbb{C}}$ of rank $r^{\prime}$ and degree $d^{\prime}$, where $r^{\prime}$ and $d^{\prime}$ are as in Proposition 6.7. As in eqn. (6.1), let

$$
\Gamma_{r^{\prime}} \subset \operatorname{Pic}^{0}\left(X_{\mathbb{C}}\right)
$$

be the subgroup of line bundles $L$ over $X_{\mathbb{C}}$ such that $L^{\otimes r^{\prime}} \cong \mathcal{O}_{X_{\mathbb{C}}}$. If we fix any $E \in \mathcal{M}_{X_{\mathbb{C}}}\left(r^{\prime}, d^{\prime}\right)$, then the map

$$
\operatorname{Pic}^{0}\left(X_{\mathbb{C}}\right) \longrightarrow \mathcal{M}_{X_{\mathbb{C}}}\left(r^{\prime}, d^{\prime}\right)
$$

defined by

$$
L \longmapsto E \otimes L
$$

induces an algebraic isomorphism of $\operatorname{Pic}^{0}\left(X_{\mathbb{C}}\right) / \Gamma_{r^{\prime}}$ with $\mathcal{M}_{X_{\mathbb{C}}}\left(r^{\prime}, d^{\prime}\right)$ (see Theorem 6.1). If we fix $E=V \otimes_{\mathbb{R}} \mathbb{C}$, where $V$ is a real algebraic vector bundle over $X$ (such vector bundles exist by Theorem 6.3), then the involution of $\mathcal{M}_{X_{\mathbb{C}}}\left(r^{\prime}, d^{\prime}\right)$ defined by $W \longrightarrow \sigma^{*} \bar{W}$ corresponds to the involution of $\operatorname{Pic}^{0}\left(X_{\mathbb{C}}\right)$ defined by $L \longrightarrow \sigma^{*} \bar{L}$; see eqn. (6.18). Therefore, Proposition 6.7 has the following corollary:

Corollary 6.8. Take $r:=2 r^{\prime}$ and $d:=2 d^{\prime}$, where $d^{\prime}$ is an odd integer and $r^{\prime}$ is a positive integer coprime to $d^{\prime}$. The set of isomorphism classes of stable real algebraic vector bundles over $X$ of rank $r$ and degree $d$ is canonically identified with the quotient space $\mathcal{M}_{X_{\mathbb{C}}}\left(r^{\prime}, d^{\prime}\right) /(\mathbb{Z} / 2 \mathbb{Z})$ for the involution of $\mathcal{M}_{X_{\mathbb{C}}}\left(r^{\prime}, d^{\prime}\right)$ defined by

$$
W \longrightarrow \sigma^{*} \bar{W}
$$

They are also isomorphic to the quotient space $\left(\operatorname{Pic}^{0}\left(X_{\mathbb{C}}\right) / \Gamma_{r^{\prime}}\right) /(\mathbb{Z} / 2 \mathbb{Z})$ for the involution of $\operatorname{Pic}^{0}\left(X_{\mathbb{C}}\right) / \Gamma_{r^{\prime}}$ defined by $L \longmapsto \sigma^{*} \bar{L}$.

We will now consider the case where $d^{\prime}$ is an even integer.

Proposition 6.9. Fix a positive integer $r^{\prime}$ and an even integer $d^{\prime}$ such that $r^{\prime}$ and $d^{\prime}$ are mutually coprime. Let $\mathcal{M}_{X_{\mathbb{C}}}\left(r^{\prime}, d^{\prime}\right) \backslash \mathcal{M}_{X}\left(r^{\prime}, d^{\prime}\right)$ denote the set of isomorphism 
classes of stable vector bundles over $X_{\mathbb{C}}$ of rank $r^{\prime}$ and degree $d^{\prime}$ which are not of the form $F \otimes_{\mathbb{R}} \mathbb{C}$, where $F$ is some real algebraic vector bundle over $X$. Set $r:=2 r^{\prime}$ and $d:=2 d^{\prime}$.

(1) For any stable vector bundle $E \in \mathcal{M}_{X_{\mathbb{C}}}\left(r^{\prime}, d^{\prime}\right) \backslash \mathcal{M}_{X}\left(r^{\prime}, d^{\prime}\right)$ over $X_{\mathbb{C}}$, the real algebraic vector bundle over $X$ defined by $E \oplus \sigma^{*} \bar{E}$ is stable.

(2) For any stable real algebraic vector bundle $V$ over $X$ of rank $r$ and degree $d$, there is a stable vector bundle $E \in \mathcal{M}_{X_{\mathbb{C}}}\left(r^{\prime}, d^{\prime}\right) \backslash \mathcal{M}_{X}\left(r^{\prime}, d^{\prime}\right)$ over $X_{\mathbb{C}}$ such that

$$
V \otimes_{\mathbb{R}} \mathbb{C}=E \oplus \sigma^{*} \bar{E}
$$

(3) Take vector bundles $V, W \in \mathcal{M}_{X_{\mathbb{C}}}\left(r^{\prime}, d^{\prime}\right) \backslash \mathcal{M}_{X}\left(r^{\prime}, d^{\prime}\right)$. Let $V_{\mathbb{R}}$ (respectively, $\left.W_{\mathbb{R}}\right)$ be the real algebraic vector bundle over $X$ given by $V \oplus \sigma^{*} \bar{V}$ (respectively, $W \oplus \sigma^{*} \bar{W}$ ). Then $V_{\mathbb{R}}$ and $W_{\mathbb{R}}$ are isomorphic if and only if either $V$ is isomorphic to $W$ or $V$ is isomorphic to $\sigma^{*} \bar{W}$.

Proof. Take any $E \in \mathcal{M}_{X_{\mathbb{C}}}\left(r^{\prime}, d^{\prime}\right) \backslash \mathcal{M}_{X}\left(r^{\prime}, d^{\prime}\right)$. Since $E \oplus \sigma^{*} \bar{E}$ is polystable, by Lemma 4.1(3), the real algebraic vector bundle $V$ over $X$ defined by $E \oplus \sigma^{*} \bar{E}$ is polystable. Assume that $V$ is not stable. So $V=V_{1} \oplus V_{2}$, where both $V_{1}$ and $V_{2}$ are nonzero real algebraic vector bundles. Since

$$
E \oplus \sigma^{*} \bar{E}=V \otimes_{\mathbb{R}} \mathbb{C}=\left(V_{1} \otimes_{\mathbb{R}} \mathbb{C}\right) \oplus\left(V_{2} \otimes_{\mathbb{R}} \mathbb{C}\right),
$$

and both $E$ and $\sigma^{*} \bar{E}$ are indecomposable, from [At1, p. 315, Theorem 2] we conclude that either $E=V_{1} \otimes_{\mathbb{R}} \mathbb{C}$ or $E=V_{2} \otimes_{\mathbb{R}} \mathbb{C}$. This contradicts the assumption that $E \in \mathcal{M}_{X_{\mathbb{C}}}\left(r^{\prime}, d^{\prime}\right) \backslash \mathcal{M}_{X}\left(r^{\prime}, d^{\prime}\right)$. Therefore, the real algebraic vector bundle $V$ is stable.

To prove the second statement, let $V$ be a stable real algebraic vector bundle over $X$ of rank $r$ and degree $d$. Then the complex vector bundle

$$
V_{\mathbb{C}}:=V \otimes_{\mathbb{R}} \mathbb{C}
$$

over $X_{\mathbb{C}}$ is polystable (see Lemma 4.1(3)).

Since $\operatorname{gcd}(r, d) \neq 1$, the polystable vector bundle $V_{\mathbb{C}}$ is not stable (see Theorem 6.4). Since $\operatorname{gcd}(r, d)=2$, we have

$$
V_{\mathbb{C}}=E_{1} \oplus E_{2}
$$

where both $E_{1}$ and $E_{2}$ are nonzero stable vector bundles.

Let $\delta: V_{\mathbb{C}} \longrightarrow \sigma^{*} \overline{V_{\mathbb{C}}}$ be the isomorphism as in eqn. (2.2). Since $V$ is stable, $\delta\left(E_{1}\right) \neq \sigma^{*} \overline{E_{1}}$. Therefore, the composition homomorphism

$$
E_{1} \stackrel{\delta}{\longrightarrow} \sigma^{*} \overline{V_{\mathbb{C}}} \longrightarrow\left(\sigma^{*} \overline{V_{\mathbb{C}}}\right) / \sigma^{*} \overline{E_{1}}=\sigma^{*} \overline{E_{2}}
$$

is nonzero. Since $E_{1}$ and $\sigma^{*} \overline{E_{2}}$ are stable with

$$
\frac{\operatorname{degree}\left(E_{1}\right)}{\operatorname{rank}\left(E_{1}\right)}=\frac{\operatorname{degree}\left(\sigma^{*} \overline{E_{2}}\right)}{\operatorname{rank}\left(\sigma^{*} \overline{E_{2}}\right)},
$$

this implies that the above composition homomorphism $E_{1} \longrightarrow \sigma^{*} \overline{E_{2}}$ is an isomorphism.

To complete the proof of the second statement we need to show that

$$
E_{1} \neq F \otimes_{\mathbb{R}} \mathbb{C}
$$


for some real algebraic vector bundle $F$ over $X$. Assume that $E_{1}=F \otimes_{\mathbb{R}} \mathbb{C}$. Then

$$
V_{\mathbb{C}}=E_{1} \oplus \sigma^{*} \overline{E_{1}}=(F \oplus F) \otimes_{\mathbb{R}} \mathbb{C} .
$$

Now from Lemma 2.1] it follows that $V=F \oplus F$. This contradicts the assumption that $V$ is stable. This completes the proof of the second statement.

The third statement follows from [At1, p. 315, Theorem 2] and Lemma 2.1] This completes the proof of the proposition.

The following corollary is deduced using Proposition 6.9, just as Corollary 6.8 is deduced from Proposition 6.7

Corollary 6.10. Take $r^{\prime}$ and $d^{\prime}$ as in Proposition 6.9. Let $\mathcal{M}_{X_{\mathbb{C}}}\left(r^{\prime}, d^{\prime}\right)$ be the moduli space of stable vector bundles over $X_{\mathbb{C}}$ of rank $r^{\prime}$ and degree $d^{\prime}$. Let

$$
\mathcal{M}_{X_{\mathbb{C}}}\left(r^{\prime}, d^{\prime}\right) \backslash \mathcal{M}_{X}\left(r^{\prime}, d^{\prime}\right) \subset \mathcal{M}_{X_{\mathbb{C}}}\left(r^{\prime}, d^{\prime}\right)
$$

denote the subset defined by all stable vector bundles which are not of the form $F \otimes_{\mathbb{R}} \mathbb{C}$, where $F$ is some real algebraic vector bundle over $X$. Then the set of all isomorphism classes of stable real algebraic vector bundles over $X$ of rank $r$ and degree $d$ is canonically identified with the quotient space $\left(\mathcal{M}_{X_{\mathbb{C}}}\left(r^{\prime}, d^{\prime}\right) \backslash \mathcal{M}_{X}\left(r^{\prime}, d^{\prime}\right)\right) /(\mathbb{Z} / 2 \mathbb{Z})$ for the involution of $\mathcal{M}_{X_{\mathbb{C}}}\left(r^{\prime}, d^{\prime}\right) \backslash \mathcal{M}_{X}\left(r^{\prime}, d^{\prime}\right)$ defined by $W \longrightarrow \sigma^{*} \bar{W}$.

Let $\operatorname{Pic}^{0}(X) \subset \operatorname{Pic}^{0}\left(X_{\mathbb{C}}\right)$ be the subset consisting of all line bundles over $X_{\mathbb{C}}$ of the form $L \otimes_{\mathbb{R}} \mathbb{C}$, where $L$ is some real algebraic line bundle over $X$. The space $\left(\mathcal{M}_{X_{\mathbb{C}}}\left(r^{\prime}, d^{\prime}\right) \backslash \mathcal{M}_{X}\left(r^{\prime}, d^{\prime}\right)\right) /(\mathbb{Z} / 2 \mathbb{Z})$ in Corollary 6.10 is isomorphic to the quotient space $\left(\left(\operatorname{Pic}^{0}\left(X_{\mathbb{C}}\right) / \Gamma_{r^{\prime}}\right) \backslash\left(\operatorname{Pic}^{0}(X) / \Gamma_{r^{\prime}}^{\mathbb{R}}\right)\right) /(\mathbb{Z} / 2 \mathbb{Z})$ for the involution of $\operatorname{Pic}^{0}\left(X_{\mathbb{C}}\right) / \Gamma_{r^{\prime}}$ defined by $L \longmapsto \sigma^{*} \bar{L}$, where $\Gamma_{r^{\prime}}$ is the group of line bundles $L$ over $X_{\mathbb{C}}$ with $L^{\otimes r^{\prime}} \cong \mathcal{O}_{X_{\mathbb{C}}}$, and $\Gamma_{r^{\prime}}^{\mathbb{R}}:=\operatorname{Pic}^{0}(X) \cap \Gamma_{r^{\prime}}$ as in eqn. (6.12).

Remark 6.11. In view of Corollary 6.6, we conclude that Theorem 6.3, Proposition 6.7 and Proposition 6.9 together classify all stable real algebraic vector bundles over $X$. Therefore, we have classified all polystable real algebraic vector bundles over $X$.

\section{ACKNOWLEDGEMENTS}

We thank the referee for helpful comments to improve the exposition.

\section{REFERENCES}

[AG] Norman L. Alling and Newcomb Greenleaf, Foundations of the theory of Klein surfaces, Lecture Notes in Mathematics, Vol. 219, Springer-Verlag, Berlin-New York, 1971. MR0333163 $(48: 11488)$

[At1] Michael F. Atiyah, On the Krull-Schmidt theorem with application to sheaves, Bull. Soc. Math. Fr. 84 (1956), 307-317. MR0086358 (19:172b)

[At2] Michael F. Atiyah, Vector bundles over an elliptic curve, Proc. Lond. Math. Soc. 7 (1957), 414-452. MR0131423 (24:A1274)

[HL] Daniel Huybrechts and Manfred Lehn, The geometry of moduli spaces of sheaves, Aspects of Mathematics, E31. Friedr. Vieweg \& Sohn, Braunschweig, 1997. MR.1450870 (98g:14012)

[Ne] Peter E. Newstead, Introduction to moduli problems and orbit spaces, T.I.F.R. Lectures on Mathematics and Physics, 51, Narosa Publishing House, New Delhi, 1978. MR546290 $(81 \mathrm{k}: 14002)$ 
[Si] Robert Silhol, Real algebraic surfaces, Lect. Notes Math. no. 1392, Springer-Verlag, Berlin Heidelberg, 1989 MR.1015720 (91i:14045)

[Tu] Loring W. Tu, Semistable bundles over an elliptic curve, Adv. Math. 98 (1993), 1-26. MR 1212625 (94a:14008)

School of Mathematics, Tata Institute of Fundamental Research, Homi Bhabha Road, Mumbai 400005, India

E-mail address: usha@math.tifr.res.in

School of Mathematics, Tata Institute of Fundamental Research, Homi Bhabha Road, Mumbai 400005, India

E-mail address: indranil@math.tifr.res.in 